\title{
COMPLEX VECTOR FIELDS AND HYPOELLIPTIC PARTIAL DIFFERENTIAL OPERATORS
}

\author{
ANDREA ALTOMANI, C. DENSON HILL, MAURO NACINOVICH, \\ AND EGMONT PORTEN
}

\begin{abstract}
We prove a subelliptic estimate for systems of complex vector fields under some assumptions that generalize the essential pseudoconcavity for $C R$ manifolds, that was first introduced by two of the Authors, and the Hörmander's bracket condition for real vector fields. Applications are given to prove the hypoellipticity of first order systems and second order partial differential operators. Finally we describe a class of compact homogeneous $C R$ manifolds for which the distribution of $(0,1)$ vector fields satisfies a subelliptic estimate.
\end{abstract}

\section{Contents}

Introduction

1. Subelliptic systems of complex vector fields

2. The distributions $\mathbb{K}_{\mathfrak{Z}}(M)$ and $\Theta_{\mathfrak{Z}}(M)$

3. Pullbacks of Distributions

4. Hypoellipticity for some differential operators of the first and of the second order

5. Applications to almost $C R$ manifolds

6. Subellipticity conditions for homogeneous $C R$ manifolds

7. Orbits of a real form in a complex flag manifold

References

\section{INTRODUCTION}

In this paper we prove a subelliptic estimate for sums of squares of complex vector fields. Namely, given a distribution $\mathfrak{Z}(M)$ of complex vector fields on an $m$ dimensional real smooth manifold $M$, we find conditions for the subellipticity of $\mathfrak{Z}(M)$, i.e. the validity of the estimate

$$
\|u\|_{\epsilon}^{2} \leq C\left(\|u\|_{0}^{2}+\sum_{j=1}^{n}\left\|L_{j}(u)\right\|_{0}^{2}\right) \quad \forall u \in \mathcal{C}_{0}^{\infty}(U),
$$

where $U$ is a relatively compact open subset of $M$ and $\epsilon>0, C>0$ are positive constants, depending on $U$ and on $L_{1}, \ldots, L_{n} \in \mathfrak{Z}(M)$, and $\mathcal{C}_{0}^{\infty}(U)$ is the space of smooth complex valued functions on $M$, with compact support

Date: November 20, 2018.

2000 Mathematics Subject Classification. Primary: 35H20 Secondary: 35H10 32V20.

Key words and phrases. Complex distribution, subelliptic estimate, hypoellipticity, Levi form, $C R$ manifold, pseudoconcavity, flag manifold. 
contained in $U$. It is known that this estimate implies the $\mathcal{C}^{\infty}$-hypoellipticity in $U$ of $\sum_{j=1}^{n} L_{j}^{*} L_{j}$ (see e.g. [16, 12]).

Our result directly applies to the study of the overdetermined systems of first order partial differential operators on $M$, that are canonically associated to $\mathfrak{Z}(M)$ and to a $\mathbb{C}$-linear connection on a complex vector bundle $E \stackrel{\pi}{\rightarrow} M$.

We also investigate the $\mathcal{C}^{\infty}$-hypoellipticity of more general second order partial differential operators on $M$, of the form

$$
P(u)=\sum_{j=1}^{n} \bar{L}_{j} L_{j}(u)+L_{0}(u)+a u,
$$

where $a$ is a complex valued smooth function in $\mathcal{C}^{\infty}(M), L_{0}, L_{1}, \ldots, L_{n}$ are smooth complex vector fields on $M$, and only the imaginary part of $L_{0}$ is required to belong to the $\mathcal{C}^{\infty}(M)$-linear span of $L_{1}, \ldots, L_{n}, \bar{L}_{1}, \ldots, \bar{L}_{n}$.

Our original motivation, and also our main applications, involve $C R$ manifolds. However, the study of (0.1) and of the operators (0.2) is of independent interest, and related questions have been considered recently in [18, 7, 6, 22, 23]. These papers show that the consideration of complex $L_{j}$ 's brings completely new phenomena, compared with the real case (see e.g. [14, 8]).

Condition (1.21) for the subellipticity of $\mathfrak{Z}(M)$ can be viewed as a generalization, at the same time, of both the essential pseudoconcavity of [12] in $C R$ geometry and Hörmander's condition of [14] for the generalized Kolmogorov equation. In fact, in $\$ 2$ we prove that, at points that are generic for $\mathfrak{Z}(M)$ (in a sense made precise in Definition 2.15), it is equivalent to a condition (2.32), that involves semidefinite generalized Levi forms attached to $\mathfrak{Z}(M)$ and their kernels. This quite explicit formulation was suggested by specific examples from [1]. However, conditions (1.21) and (2.32) apply to more general contexts than $C R$ geometry. At the beginning, we assume neither that $\mathfrak{Z}(M)$ is a distribution of constant rank, nor that it satisfies any formal integrability condition, nor anything special about the intersection $\mathfrak{Z}(M) \cap \overline{\mathfrak{Z}(M)}$; but conditions of this type need to be imposed in $₫ 2$ to obtain the equivalence of (1.21) and (2.32).

The work of [18, 7, 6] shows that the condition that the Lie algebra generated by $\mathfrak{Z}(M)$ spans the full complexified tangent space is in general not sufficient either for subellipticity or for the $\mathcal{C}^{\infty}$-hypoellipticity of the sum of squares. The present work is complementary, inasmuch as our sharpest results hold away from singularities and in the case where $\mathfrak{Z}(M)$ is a Lie algebra.

We reduce the question of the subellipticity of $\mathfrak{Z}(M)$ to one involving real vector fields. Indeed, our task is to find all real vector fields $X$ that are enthralled by $\mathfrak{Z}(M)$, i.e. satisfy

$$
\|X(u)\|_{\epsilon-1}^{2} \leq C\left(\sum_{j=1}^{n}\left\|L_{j}(u)\right\|_{0}^{2}+\|u\|_{0}^{2}\right), \quad \forall u \in \mathcal{C}_{0}^{\infty}(U)
$$

for some $L_{1}, \ldots, L_{n} \in \mathfrak{Z}(M)$ and $\epsilon>0, C>0$ depending on $X$ and $U$. In $\$ 1$ we observe that this set is a module over the Lie algebra $\mathfrak{A}_{\mathfrak{Z}}(M)$ generated by those real vector fields $X$ for which (0.3) holds with $\epsilon=1$. This was 
essentially shown in [16. By an argument of J.J.Kohn (see 18 and Lemma 1.8 below), we know that $(\operatorname{Re} Z)$ satisfies (0.3) with $\epsilon=\frac{1}{2}$ for all $Z \in \mathfrak{Z}(M)$. On the other hand, on an open dense subset $M^{\prime}$ of $M$ that excludes some singular points for $\mathfrak{Z}(M)$, one can check that a real vector field $X$ satisfying (0.3) with $\epsilon>\frac{1}{2}$ is necessarily the real part of some $Z \in \mathfrak{Z}(M)$. Hence we conjecture that the real vector fields $X$, satisfying (0.3), coincide on a dense open subset of $M$ with the elements of the $\mathfrak{A}_{\mathfrak{Z}}(M)$-module generated by the real parts of the elements of $\mathfrak{Z}(M)$. In $\$ 2$ we characterize, outside the singular set, the Lie algebra $\mathfrak{A}_{\mathfrak{Z}}(M)$. Then equality (2.31), together with Lemma 1.8, would give a complete and explicit description of the set of real vector fields enthralled by $\mathfrak{Z}(M)$.

A motivation for [12] was to understand the structure of a large class of $C R$ manifolds, of higher $C R$ codimension, that are of finite type in the sense of [4], and are not pseudoconcave in the sense of [11], because of the vanishing of their scalar Levi forms in some characteristic codirections. To prove also in this case a subelliptic estimate for the tangential Cauchy-Riemann operator on functions, two of the authors invented in [12] the notion of essential pseudoconcavity. Compared with the usual more restrictive notion of pseudoconcavity, it allows some of the scalar Levi forms to be zero. The subelliptic estimate is then obtained for weaker Sobolev norms than the $\frac{1}{2}$-norm in [11]. On essentially pseudoconcave almost $C R$ manifolds the maximum modulus principle and the weak unique continuation principle for $C R$ functions ([12, 13]) are valid. Under the additional assumption of formal integrability of the $C R$ structure, it was also possible to prove, in case the $C R$ manifold is either compact or real analytic, finiteness and vanishing theorems for the highest cohomology groups of the $\bar{\partial}_{M}$-complexes ([21]).

Our new condition is more general than the weak pseudoconcavity of [12], as here we allow the scalar Levi forms in some characteristic codirections to be semidefinite. As a heuristic motivation, consider a $C R$ submanifold $M$ of a complex manifold $F$. If $M$ is contained in a strictly pseudoconvex real hypersurface of $F$, then it is easy to find $L_{\mathrm{loc}}^{2}$ germs of $C R$ functions that are not smooth. We can expect, vice versa, that all $C R$ distributions on $M$ are smooth when all germs of hypersurfaces through $M$ in $F$ are strictly pseudoconcave. This is indeed the case when our condition (2.32) is verified. It is discussed in $\$ 5$.

As in [12, the homogeneous examples have strongly inspired our investigation. After considering general homogeneous $C R$ manifolds in $\$ 6$, in $\$ 7$ we classify all minimal orbits of real forms $\mathbf{G}$ of $\mathbf{G}^{\mathbb{C}}$-homogeneous flag manifolds (see e.g. [24, 1, 2]) that enjoy condition (1.21). In [1, §13], together with Prof. Medori, two of the authors gave the complete classification of the essentially pseudoconcave minimal orbits. Here we show that those satisfying (1.21) form a strictly larger class of $C R$ manifolds, on which the local $C R$ distributions are smooth.

We collected our results on hypoellipticity in $\$ 4$. Given a complex vector bundle $E \stackrel{\pi}{\rightarrow} M$, endowed with a $\mathbb{C}$-linear connection $\nabla$, for each complex vector field $Z$ on $M$ we obtain a differential operator $\nabla_{Z}$, acting on the sections of $E$. We prove that the subellipticity of $\mathfrak{Z}(M)$ implies the 
$\mathcal{C}^{\infty}$-hypoellipticity of $\left(\nabla_{Z}\right)_{Z \in \mathcal{Z}(M)}$. This yields, for a compact $M$, finite dimensionality of the space of global solutions of $\left(\nabla_{Z}(u)\right)_{Z \in \mathfrak{Z}(M)}=0$, and closedness of the range of $\left(\nabla_{Z}\right)_{Z \in \mathfrak{Z}(M)}$. For $C R$ manifolds, this implies that the cohomology groups $H_{\bar{\partial}_{M}}^{p, 1}(M)$ of the tangential Cauchy-Riemann complex are Hausdorff.

The subellipticity of $\mathfrak{Z}(M)$ gives $\mathcal{C}^{\infty}$-hypoellipticity for the sum of squares and also for slightly more general operators (see Theorem 4.3). The question of generalizing the Kolmogorov equation to the case of complex vector fields is more complicated. We obtained two different formulations in Theorems 4.4 and 4.8. In the former, we obtain $\mathcal{C}^{\infty}$-hypoellipticity under the condition that the $\mathfrak{A}_{\mathfrak{Z}}(M)$-module generated by the real parts of the vector fields in $\mathfrak{Z}(M)$ and the "time" vector field span the whole tangent space at a point. This distinction between "space" and "time" vector fields makes this result weaker than the one of [14] for the case $\mathfrak{Z}(M)=\overline{\mathfrak{Z}(M)}$. This is the reason we prove in Theorem 4.8 a result generalizing [14], under the condition that the real parts of the vector fields in $\mathfrak{Z}(M)$ satisfy (0.3) with $\epsilon=1$.

\section{Subelliptic Systems of COMPlex Vector Fields}

Let $M$ be a smooth real manifold of dimension $m$. For $U$ open in $M$, we denote by $\mathcal{C}^{\infty}(U)$ (resp. $\mathcal{C}_{0}^{\infty}(U)$ ) the space of complex valued smooth functions (resp. with compact support) in $U$, and by $\mathfrak{X}(U)$ (resp. $\mathfrak{X}^{\mathbb{C}}(U)$ ) the Lie algebra of the smooth real (resp. complex) vector fields in $U$.

Definition 1.1. A distribution of complex vector fields $\mathfrak{Z}(M)$ in $M$ is any $\mathcal{C}^{\infty}(M)$-left submodule of $\mathfrak{X}^{\mathbb{C}}(M)$. This means that $Z_{1}+Z_{2}$ and $f Z$ belong to $\mathfrak{Z}(M)$ if $Z_{1}, Z_{2}, Z \in \mathfrak{Z}(M)$ and $f \in \mathcal{C}^{\infty}(M)$.

For each point $p \in M$ we set

$$
Z_{p} M=\{Z(p) \mid Z \in \mathfrak{Z}(M)\} \subset T_{p}^{\mathbb{C}} M=\mathbb{C} \otimes_{\mathbb{R}} T_{p} M .
$$

The dimension of the complex vector space $Z_{p} M$ is called the rank of $\mathfrak{Z}(M)$ at $p$. We do not assume in the definition that $\mathfrak{Z}(M)$ has a constant rank. The points where the rank of $\mathfrak{Z}(M)$ is not constant on a neighborhood are the singularities of $\mathfrak{Z}(M)$. If $\mathfrak{Z}(M)=\left\{Z \in \mathfrak{X}^{\mathbb{C}}(M) \mid Z(p) \in Z_{p} M, \forall p \in M\right\}$, we say that $\mathfrak{Z}(M)$ has at most simple singularities.

We say that $\mathfrak{Z}(M)$ is formally integrable if

$$
[\mathfrak{Z}(M), \mathfrak{Z}(M)] \subset \mathfrak{Z}(M) .
$$

Distributions of real vector fields and their singular points are defined likewise.

Definition 1.2. The distribution $\mathfrak{Z}(M)$ is said to be subelliptic at $p \in M$ if there is an open neighborhood $U$ of $p$ in $M$, a real $\epsilon>0$, a constant $C>0$, and a finite set $L_{1}, \ldots, L_{n}$ of vector fields from $\mathfrak{Z}(M)$, such that (0.1) is satisfied, where $\|\cdot\|_{\epsilon}$ and $\|\cdot\|_{0}$ are the $\epsilon$-Sobolev norms and the $L^{2}$-norm with respect to some Riemannian metric on $M$ (see e.g. [9]).

Remark 1.3. When $\mathfrak{Z}(M)$ is the complexification of a distribution of real vector fields $\mathfrak{Y}(M) \subset \mathfrak{X}(M)$, then (0.1), at a generic point $p \in M$, is equivalent to the fact that $\mathfrak{Z}(M)$ and its higher order commutators span the whole complexified tangent space $T_{p}^{\mathbb{C}} M$ (see, e.g. [14, 8]). However this condition 
is neither necessary nor sufficient, and does not imply $\mathcal{C}^{\infty}$-hypoellipticity of the associated sum of squares operators when the vector fields are complex (cf. [18, 7, 6, 23]).

Definition 1.4. We say that $\mathfrak{Z}(M)$ enthralls a vector field $Z \in \mathfrak{X}^{\mathbb{C}}(M)$ if

$$
\left\{\begin{array}{c}
\forall U^{\text {open }} \Subset M, \exists L_{1}, \ldots, L_{n} \in \mathfrak{Z}(M), \exists \epsilon>0, C>0 \text { s.t. } \\
\|Z(u)\|_{\epsilon-1}^{2} \leq C\left(\sum_{j=1}^{n}\left\|L_{j}(u)\right\|_{0}^{2}+\|u\|_{0}^{2}\right), \quad \forall u \in \mathcal{C}_{0}^{\infty}(U) .
\end{array}\right.
$$

Set

$$
\mathbb{S}_{\mathfrak{Z}}(M)=\left\{Z \in \mathfrak{X}^{\mathbb{C}}(M) \mid \mathfrak{Z}(M) \text { enthralls } Z\right\} .
$$

We notice that $\mathbb{S}_{\mathfrak{Z}}(M)$ is a distribution of complex vector fields containing $\mathfrak{Z}(M)$, and $\mathbb{S}_{\mathfrak{Z}}(M) \cap \mathfrak{X}(M)$ is a distribution of real vector fields, and that both are spaces of global sections of fine sheaves of left modules, the first over the sheaf of germs of complex valued smooth functions, the second over the sheaf of germs of real valued smooth functions on $M$.

By the real Frobenius theorem we obtain (see e.g. [14])

Proposition 1.5. Let $\mathfrak{W}_{\mathfrak{Z}}(M)$ be the Lie subalgebra of $\mathfrak{X}^{\mathbb{C}}(M)$ generated by $\mathfrak{Z}(M)+\overline{\mathfrak{Z}(M)}$. Then, if $\mathfrak{W}_{\mathfrak{Z}}(M)$ is a distribution of constant rank on $M$, we have

$$
\mathbb{S}_{\mathfrak{Z}}(M) \cap \mathfrak{X}(M) \subset \mathbb{S}_{\mathfrak{Z}}(M) \subset \mathfrak{W}_{\mathfrak{Z}}(M) .
$$

Remark 1.6. The complex distribution $\mathfrak{Z}(M)$ is subelliptic at $p \in M$ if and only if

$$
\left\{X(p) \mid X \in \mathbb{S}_{\mathfrak{Z}}(M) \cap \mathfrak{X}(M)\right\}=T_{p} M .
$$

Remark 1.7. If $Z \in \mathbb{S}_{\mathfrak{Z}}(M)$ and $\Lambda_{-1}$ is a properly supported pseudodifferential operator of order $(-1)$ having its symbol in the class $S_{1,0}^{-1}$, then $\Lambda_{-1} \circ Z$ is a subelliptic multiplier for $\mathfrak{Z}(M)$ in the sense of J.J.Kohn (see e.g. [17]).

Pseudodifferential operators will be an important tool in the following. For their definition and properties we refer to [15, Chapter XVIII].

If $U$ is an open subset of $M$, and $s$ a real number, we shall denote by $\Psi^{s}(U)$ the space of properly supported pseudodifferential operators in $U$, of order less or equal than $s$, with symbol in $S_{1,0}^{s}$. For each coordinate neighborhood $V \subset U$, a $\Lambda \in \Psi^{s}(U)$ is defined, in the local coordinates, by

$$
\Lambda(u)=\iint_{U \times \mathbb{R}^{m}} e^{i\langle x-y, \xi\rangle} a(x, \xi) u(y) d y d \xi, \quad \text { for } u \in \mathcal{C}_{0}^{\infty}(V),
$$

with $a \in \mathcal{C}^{\infty}\left(V \times \mathbb{R}^{m}\right)$ and

$$
\left\{\begin{array}{c}
\forall K \Subset V, \forall \alpha, \beta \in \mathbb{N}^{m}, \exists C=C(K, \alpha, \beta)>0 \text { s.t. } \\
\left|D_{x}^{\alpha} D_{\xi}^{\beta} a(x, \xi)\right| \leq C(1+|\xi|)^{s-|\beta|}, \forall(x, \xi) \in K \times \mathbb{R}^{m} .
\end{array}\right.
$$

The fact that $\Lambda \in \Psi^{s}(U)$ is properly supported means that for every $K \Subset U$ there is $K^{\prime} \Subset U$ such that

$$
\operatorname{supp}(u) \subset K \Rightarrow \operatorname{supp}(\Lambda(u)) \subset K^{\prime} .
$$


The following Lemma is essentially contained in [18, p.949].

Lemma 1.8. Let $Z \in \mathfrak{X}^{\mathbb{C}}(M)$ be any complex vector field. For every relatively compact open subset $U \Subset M$ there is a constant $C>0$ such that

$$
\|\bar{Z}(u)\|_{-\frac{1}{2}}^{2} \leq C\left(\|Z(u)\|_{0}^{2}+\|u\|_{0}^{2}\right) \quad \forall u \in \mathcal{C}_{0}^{\infty}(U) .
$$

Hence

$$
\begin{gathered}
\mathfrak{Z}(M)+\overline{\mathfrak{Z}(M)} \subset \mathbb{S}_{\mathfrak{Z}}(M), \\
\{Z+\bar{Z} \mid Z \in \mathfrak{Z}(M)\} \subset \mathbb{S}_{\mathfrak{Z}}(M) \cap \mathfrak{X}(M) .
\end{gathered}
$$

Proof. Let $Z \in \mathfrak{Z}(M)$ and $U^{\text {open }} \Subset M$. Then, with $\Lambda_{0} \in \Psi^{0}(U)$ and constants $C_{1}, C_{2}>0$, we obtain

$$
\begin{aligned}
\|\bar{Z}(u)\|_{-\frac{1}{2}}^{2}=\left(\bar{Z}(u) \mid \Lambda_{0}(u)\right)_{0} & \leq\left|\left(\Lambda_{0}^{*}(u) \mid Z(u)\right)_{0}\right|+C_{1}\|u\|_{0}^{2} \\
& \leq C_{2}\left(\|Z(u)\|_{0}+\|u\|_{0}\right)\|u\|_{0}, \quad \forall u \in \mathcal{C}^{\infty}(U) .
\end{aligned}
$$

This yields (1.10), and hence also (1.11) and (1.12).

In particular, if $\mathfrak{Z}(M) \neq\{0\}$, the distribution $\mathbb{S}_{\mathfrak{Z}}(M) \cap \mathfrak{X}(M)$ of real vector fields that are enthralled by $\mathfrak{Z}(M)$ is not trivial.

Definition 1.9. We denote by $\mathbb{E}_{\mathfrak{Z}}(M)$ the set of $Z \in \mathfrak{X}^{\mathbb{C}}(M)$ such that

$$
\left\{\begin{array}{c}
\forall U^{\text {open }} \Subset M, \exists L_{1}, \ldots, L_{n} \in \mathfrak{Z}(M), \exists C>0 \text { s.t } \\
\|Z(u)\|_{0}^{2} \leq C\left(\sum_{j=1}^{n}\left\|L_{j}(u)\right\|_{0}^{2}+\|u\|_{0}^{2}\right), \quad \forall u \in \mathcal{C}_{0}^{\infty}(U) .
\end{array}\right.
$$

Also $\mathbb{E}_{\mathfrak{Z}}(M)$ is a distribution of complex vector fields, with

$$
\mathfrak{Z}(M) \subset \mathbb{E}_{\mathfrak{Z}}(M) \subset \mathbb{S}_{\mathfrak{Z}}(M) .
$$

As a consequence of Lemma 1.8, we get

\section{Lemma 1.10.}

$$
\mathbb{E}_{\mathfrak{Z}}(M)+\overline{\mathbb{E}_{\mathfrak{Z}}(M)} \subset \mathbb{S}_{\mathfrak{Z}}(M) .
$$

Definition 1.11. Set

(1.18) $\mathbb{T}_{\mathfrak{Z}}^{(0)}(M)=\{Z+\bar{Z} \mid Z \in \mathfrak{Z}(M)\}$,

$$
\begin{aligned}
& \mathbb{A}_{\mathfrak{Z}}(M)=\mathbb{E}_{\mathfrak{Z}}(M) \cap \mathfrak{X}(M), \\
& \left.\left.\mathbb{T}_{\mathfrak{Z}}^{(h)}(M)=\langle[X, Y]| X \in \mathbb{A}_{\mathfrak{Z}}(M), Y \in \mathbb{T}_{\mathfrak{Z}}^{(h-1)}(M)\right\}\right\rangle, \quad \text { for } h \geq 1, \\
& \mathfrak{T}_{\mathfrak{Z}}(M)=\sum_{h=0}^{\infty} \mathbb{T}_{\mathfrak{Z}}^{(h)}(M) .
\end{aligned}
$$

We shall consider the condition at $p \in M$ :

$$
\left\{X(p) \mid X \in \mathfrak{T}_{\mathfrak{Z}}(M)\right\}=T_{p} M .
$$

Remark 1.12. If condition (1.21) is satisfied at a point $p_{0} \in M$, then it is also satisfied at all points $p$ in an open neighborhood $U$ of $p_{0}$. 
It is convenient to introduce the notation $\left[Z_{1}, \ldots, Z_{m}\right]$ for the higher order commutator of smooth real or complex vector fields. It is recursively defined by

$$
\left\{\begin{array}{l}
{\left[Z_{1}\right]=Z_{1}} \\
{\left[Z_{1}, Z_{2}\right]=Z_{1} Z_{2}-Z_{2} Z_{1},} \\
{\left[Z_{1}, Z_{2}, \ldots, Z_{m}\right]=\left[Z_{1},\left[Z_{2}, \ldots, Z_{m}\right]\right] \text { for } m \geq 3}
\end{array}\right.
$$

Proposition 1.13. The distribution of real vector fields $\mathfrak{T}_{\mathfrak{Z}}(M)$ is an $\mathfrak{A}_{\mathfrak{Z}}(M)$ Lie-module.

Proof. We prove by recurrence on $r \geq 1$ that, if $X_{1}, \ldots, X_{r} \in \mathbb{A}_{\mathfrak{Z}}(M)$ and $Y \in \mathfrak{T}_{\mathfrak{Z}}(M)$, then also $\left[\left[X_{1}, \ldots, X_{r}\right], Y\right] \in \mathfrak{T}_{\mathfrak{Z}}(M)$. This follows from the definition of $\mathfrak{T}_{\mathfrak{Z}}(M)$ for $r=1$. Assume now that $r>1$ and that $\mathfrak{T}_{\mathfrak{Z}}(M)$ contains all commutators $\left[\left[X_{1}, \ldots, X_{r-1}\right], Y\right]$ with $X_{1}, \ldots, X_{r-1} \in \mathbb{A}_{\mathfrak{Z}}(M)$ and $Y \in \mathfrak{T}_{\mathfrak{Z}}(M)$. If $X_{1}, \ldots, X_{r} \in \mathbb{A}_{\mathfrak{Z}}(M)$ and $Y \in \mathfrak{T}_{\mathfrak{Z}}(M)$, we obtain

$$
\left[\left[X_{1}, \ldots, X_{r}\right], Y\right]=-\left[\left[X_{2}, \ldots, X_{r}\right],\left[X_{1}, Y\right]\right]+\left[X_{1},\left[\left[X_{2}, \ldots, X_{r}\right], Y\right]\right] .
$$

Since $\left[X_{1}, Y\right] \in \mathfrak{T}_{\mathfrak{Z}}(M)$, by our inductive assumption the first summand on the right hand side also belongs to $\mathfrak{T}_{\mathfrak{Z}}(M)$. By the inductive assumption, the commutator $\left[\left[X_{2}, \ldots, X_{r}\right], Y\right]$ belongs to $\mathfrak{T}_{\mathfrak{Z}}(M)$, and hence also the second summand in the right hand side belongs to $\mathfrak{T}_{\mathfrak{Z}}(M)$. The proof is complete.

Proposition 1.14. The Lie algebra of real vector fields $\mathfrak{A}_{\mathfrak{Z}}(M)$ is contained in $\mathbb{S}_{\mathfrak{Z}}(M) \cap \mathfrak{X}(M)$, and $\mathbb{S}_{\mathfrak{Z}}(M) \cap \mathfrak{X}(M)$ is an $\mathfrak{A}_{\mathfrak{Z}}(M)$-Lie-submodule of $\mathfrak{X}(M)$. In particular, we have the inclusion

$$
\mathfrak{T}_{\mathfrak{Z}}(M) \subset \mathbb{S}_{\mathfrak{Z}}(M) \cap \mathfrak{X}(M) .
$$

Proof. Let $U$ be a relatively compact open subset of $M$. Assume that $X \in$ $\mathbb{A}_{\mathfrak{Z}}(M), Y \in \mathbb{S}_{\mathfrak{Z}}(M) \cap \mathfrak{X}(M)$ and let $\epsilon>0$ be such that that $Y=Z$ satisfies the estimate in (1.3). We can assume that $0<\epsilon \leq \frac{1}{2}$. If $U^{\prime}$ is an open relatively compact subset of $U$, we have, with some $\Lambda_{\epsilon-1} \in \Psi^{\epsilon-1}(U)$ and suitable positive constants $\mathrm{C}_{0}, \mathrm{C}_{1}$,

$$
\begin{aligned}
\|[X, Y](u)\|_{\frac{\epsilon}{2}-1}^{2} & \leq\left|\left([X, Y](u) \mid \Lambda_{\epsilon-1}(u)\right)_{0}\right| \\
& \leq\left|\left(X Y(u) \mid \Lambda_{\epsilon-1}(u)\right)_{0}\right|+\left|\left(Y X(u) \mid \Lambda_{\epsilon-1}(u)\right)_{0}\right| \\
& \leq\left|\left(\Lambda_{\epsilon-1}^{*}(Y(u)) \mid X(u)\right)_{0}\right|+\left|\left(X(u) \mid \Lambda_{\epsilon-1} Y(u)\right)\right| \\
& \quad+\mathrm{C}_{0}\|u\|\left(\|u\|_{0}+\|Y(u)\|_{\epsilon-1}+\|X(u)\|_{0}\right) \\
& \leq \mathrm{C}_{1}\left(\|X(u)\|_{0}^{2}+\|Y(u)\|_{\epsilon-1}^{2}+\|u\|_{0}^{2}\right), \quad \forall u \in \mathcal{C}^{\infty}\left(U^{\prime}\right)
\end{aligned}
$$

The last term of this chain of inequalities is bounded by a constant times $\left(\sum_{j=1}^{n}\left\|L_{j}(u)\right\|^{2}+\|u\|_{0}^{2}\right)$, for a suitable choice of $L_{1}, \ldots, L_{n} \in \mathfrak{Z}(M)$. This shows that $[X, Y] \in \mathbb{S}_{\mathfrak{Z}}(M) \cap \mathfrak{X}(M)$.

Since $\mathbb{A}_{\mathfrak{Z}}(M) \subset \mathbb{S}_{\mathfrak{Z}}(M) \cap \mathfrak{X}(M)$, also $\mathfrak{A}_{\mathfrak{Z}}(M) \subset \mathbb{S}_{\mathfrak{Z}}(M) \cap \mathfrak{X}(M)$. The argument in the proof of Proposition 1.13 shows that, since $\left[\mathbb{A}_{\mathfrak{Z}}(M), \mathbb{S}_{\mathfrak{Z}}(M) \cap\right.$ $\mathfrak{X}(M)] \subset \mathbb{S}_{\mathfrak{Z}}(M) \cap \mathfrak{X}(M)$, this distribution is an $\mathfrak{A}_{\mathfrak{Z}}(M)$-Lie-submodule of $\mathfrak{X}(M)$. Then the inclusion (1.23) is a consequence of the inclusion (1.12).

By using Proposition 1.14 we obtain 
Corollary 1.15. Let $\mathfrak{Z}(M)$ be a smooth distribution of complex vector fields on $M$. Then $\mathfrak{Z}(M)$ is subelliptic at all points $p \in M$ at which condition (1.21) of Definition 1.11 is satisfied.

Corollary 1.15 is a trivial consequence of the inclusion (1.23). However, in $\$ 2$ we will show that actually we are able, in case $\mathfrak{Z}(M)$ is formally integrable, to compute explicitly the left hand side of (1.21) at the points of an open dense subset of $M$, where $\mathfrak{Z}(M)$ satisfies some genericity assumptions.

Remark 1.16. When $\mathfrak{Z}(M)$ is the complexification of a distribution of real vector fields, then $\mathfrak{A}_{\mathfrak{Z}}(M)=\mathfrak{T}_{\mathfrak{Z}}(M)$, and condition (1.21) is equivalent to Hörmander's condition in [14. Thus Corollary 1.15 can be viewed as a generalization of the analogous result for distributions of real vector fields.

Remark 1.17. If $\mathfrak{Z}(M)$ is the distribution of $(0,1)$-vector fields of an almost $C R$ manifold $M$, it follows from 92 below that the essential pseudoconcavity condition of 12 implies that $\mathbb{E}_{\mathfrak{Z}}(M)=\mathfrak{Z}(M)+\overline{\mathfrak{Z}(M)}$ and that $M$ is of finite type in the sense of [4]. Therefore, Corollary 1.15 also generalizes [12, Theorem 4.1].

\section{The Distributions $\mathbb{K}_{\mathfrak{Z}}(M)$ and $\Theta_{\mathfrak{Z}}(M)$}

As pointed out after the statement of Corollary 1.15, condition (1.21) becomes an effective criterion for subellipticity when it is possible to give an explicit description of $\mathbb{E}_{\mathfrak{Z}}(M)$, or of some nontrivial part of it. We begin by giving an upper bound for $\mathbb{E}_{\mathfrak{Z}}(M)$.

Lemma 2.1. Let $\mathfrak{Z}(M)$ be a distribution of complex vector fields.

If $(\mathfrak{Z}(M)+\overline{\mathfrak{Z}(M)})$ has at most simple singularities, and in particular if $(\mathfrak{Z}(M)+\overline{\mathfrak{Z}(M)})$ has constant rank, then

$$
\mathbb{E}_{\mathfrak{Z}}(M) \subset \mathfrak{Z}(M)+\overline{\mathfrak{Z}(M)} .
$$

Proof. Since $\mathbb{E}_{\mathfrak{Z}}(M) \subset \mathbb{E}_{\mathfrak{Z}+\overline{\mathfrak{Z}}}(M)$, we can reduce the proof to the case where $\mathfrak{Z}(M)=\mathfrak{Z}(M)+\overline{\mathfrak{Z}(M)}$ is the complexification of a distribution of real vector fields and $Z \in \mathbb{E}_{\mathfrak{Z}}(M)$ is real. Fix $p \in M$ and take a coordinate patch $U$ of $p$ in $M$, centered at $p$, for which (1.13) holds, for real $L_{1}, \ldots, L_{n} \in$ $\mathfrak{X}(M) \cap \mathfrak{Z}(M)$ such that $L_{1}(p), \ldots, L_{n}(p)$ generate $Z_{p} M$. We apply the inequality in (1.13) to the test function $u_{\tau}(x)=\tau^{\frac{m-4}{4}} \chi(x) e^{i \tau\langle x, \xi\rangle-(\tau / 2)|x|^{2}}$, where $\chi(x) \in \mathcal{C}_{0}^{\infty}(U)$ satisfies $\chi(x)=1$ for $x$ in a neighborhood of 0 . Denote by $z(x, \xi)$ and $\ell_{j}(x, \xi)$ the symbols of $Z$ and $L_{j}$, respectively. We obtain

$$
L_{j}\left(u_{\tau}\right)=\tau^{\frac{m-4}{4}}\left(\tau \ell_{j}(x, \xi+i x)+L_{j}(\chi)\right) e^{i \tau\langle x, \xi\rangle-(\tau / 2)|x|^{2}} .
$$

Computing the integral by the change of variables $y=x \sqrt{\tau}$, we obtain

$$
\left\|L_{j}\left(u_{\tau}\right)\right\|_{0}^{2}=\int \chi^{2}(y / \sqrt{\tau})\left|\ell_{j}(y / \sqrt{\tau}, i \xi+y / \sqrt{\tau})\right|^{2} e^{-|y|^{2}} d y+O\left(\tau^{-\infty}\right) .
$$

Likewise, we have

$$
\left\|Z\left(u_{\tau}\right)\right\|_{0}^{2}=\int \chi^{2}(y / \sqrt{\tau})|z(y / \sqrt{\tau}, i \xi+y / \sqrt{\tau})|^{2} e^{-|y|^{2}} d y+O\left(\tau^{-\infty}\right) .
$$


By letting $\tau$ tend to $\infty$ in the estimate (1.13), we obtain that

$$
|z(0, \xi)|^{2} \leq C \sum_{j=1}^{n}\left|\ell_{j}(0, \xi)\right|^{2}, \quad \forall \xi \in \mathbb{R}^{m} .
$$

Since $L_{1}, \ldots, L_{n}$ are real, the above inequality implies that $Z(p) \in Z_{p} M$. Since $p$ was an arbitrary point of $M$, this implies that $Z \in \mathfrak{Z}(M)$.

Remark 2.2. The proof of Lemma 2.1 yields a stronger statement:

Let $\mathfrak{Z}(M)$ be a distribution of complex vector fields and assume that $\mathfrak{Z}(M)+\overline{\mathfrak{Z}(M)}$ has at most simple singularities. If $Z \in \mathbb{S}_{\mathfrak{Z}}(M)$ and

$$
\left\{\begin{array}{l}
\forall U^{\text {open }} \Subset M, \quad \exists \epsilon>\frac{1}{2}, \exists L_{1}, \ldots, L_{n} \in \mathfrak{Z}(M), \exists C>0 \text { s.t. } \\
\|Z(u)\|_{\epsilon-1}^{2} \leq C\left(\sum_{j=1}^{n}\left\|L_{j}(u)\right\|_{0}^{2}+\|u\|_{0}^{2}\right), \quad \forall u \in \mathcal{C}_{0}^{\infty}(U),
\end{array}\right.
$$

then $Z \in \mathfrak{Z}(M)+\overline{\mathfrak{Z}(M)}$.

It suffices indeed to apply (2.2), in a coordinate patch $U$, as in the proof of Lemma 2.1, to the test functions $v_{\tau}=\tau^{\left(1-\epsilon^{\prime}\right) / 2} u_{\tau}$, with $\frac{1}{2}<\epsilon^{\prime}<\epsilon$, and let $\tau \rightarrow+\infty$. Then, if $\ell_{j}(0, \xi)=0$ for $j=1, \ldots, n$, the right hand side of (2.2) stays bounded, while the left hand side tends to $+\infty$, unless $z(p, \xi)=0$.

2.1. The distribution $\Theta_{3}(M)$. Lemma 2.1 suggests that, in order to find non trivial elements of $\mathbb{E}_{\mathfrak{Z}}(M)$, one should search in $\overline{\mathfrak{Z}(M)}$. To this aim, we introduce the following

Definition 2.3. Given a distribution $\mathfrak{Z}(M)$ of complex vector fields, we set

$$
\Theta_{\mathfrak{Z}}(M)=\left\{\begin{array}{l|l}
Z \in \mathfrak{Z}(M) \mid \begin{array}{c}
\exists r \geq 0, \exists Z_{1}, \ldots, Z_{r} \in \mathfrak{Z}(M), \text { s.t. } \\
{[Z, \bar{Z}]+\sum_{j=1}^{r}\left[Z_{j}, \bar{Z}_{j}\right] \in \mathfrak{Z}(M)+\overline{\mathfrak{Z}(M)}}
\end{array}
\end{array}\right.
$$

Lemma 2.4. The set $\Theta_{\mathfrak{Z}}(M)$ is a distribution of complex vector fields.

Proof. Clearly, if $Z \in \Theta_{\mathfrak{Z}}(M)$ and $\phi \in \mathcal{E}(M)$, the product $\phi Z$ also belongs to $\Theta_{\mathfrak{Z}}(M)$. To prove that $\Theta_{\mathfrak{Z}}(M)$ contains the finite sums of its elements, it suffices to show that, if, for some $r \geq 1, Z_{0}, \ldots, Z_{r} \in \Theta_{\mathfrak{Z}}(M)$ and $\sum_{j=0}^{r}\left[Z_{j}, \bar{Z}_{j}\right] \in \mathfrak{Z}(M)+\overline{\mathfrak{Z}(M)}$, then also $Z_{0}+Z_{1} \in \Theta_{\mathfrak{Z}}(M)$. This follows from: $\quad\left[Z_{0}+Z_{1}, \overline{Z_{0}+Z_{1}}\right]+\left[Z_{0}-Z_{1}, \overline{Z_{0}-Z_{1}}\right]=2\left(\left[Z_{0}, \bar{Z}_{0}\right]+\left[Z_{1}, \bar{Z}_{1}\right]\right)$.

Lemma 2.5. Let $\mathfrak{Z}(M)$ be a distribution of complex vector fields and let $\Theta_{\mathfrak{Z}}(M)$ be defined by (2.3). Then

$$
\overline{\Theta_{\mathfrak{Z}}(M)} \subset \mathbb{E}_{\mathfrak{Z}}(M) .
$$

In particular, if $Z \in \Theta_{\mathfrak{Z}}(M)$, then $Z+\bar{Z} \in \mathbb{A}_{\mathfrak{Z}}(M)$.

Proof. The proof closely follows that of [12, Theorem 2.5]. If $Z \in \Theta_{\mathfrak{Z}}(M)$, by (2.3), there are $Z_{1}, \ldots, Z_{r}, Z_{r+1} \in \mathfrak{Z}(M)$ such that

$$
[Z, \bar{Z}]+\sum_{j=1}^{r}\left[Z_{j}, \bar{Z}_{j}\right]=Z_{r+1}-\bar{Z}_{r+1} .
$$


Set $Z_{0}=Z$ and let $Z_{j}^{*}=-\bar{Z}_{j}+a_{j}$, with $a_{j} \in \mathcal{C}^{\infty}(M)$, the $L^{2}$ formal adjoint of $Z_{j}$, for $0 \leq j \leq r+1$. Integrating by parts, and using (2.5) to compute the sum of the commutators, we obtain, for all $u \in \mathcal{C}_{0}^{\infty}(M)$,

$$
\begin{aligned}
\sum_{j=0}^{r}\left\|\bar{Z}_{j}(u)\right\|_{0}^{2} & =-\sum_{j=0}^{r}\left(\left(Z_{j}-\bar{a}_{j}\right)\left(\bar{Z}_{j}(u)\right) \mid u\right)_{0} \\
& =-\sum_{j=0}^{r}\left\{\left(\left[Z_{j}, \bar{Z}_{j}\right](u) \mid u\right)_{0}+\left(\bar{Z}_{j} Z_{j}(u) \mid u\right)_{0}-\left(\bar{Z}_{j}(u) \mid a_{j} u\right)_{0}\right\} \\
& =\sum_{j=0}^{r}\left\|Z_{j}(u)\right\|_{0}^{2}+\sum_{j=0}^{r+1} \operatorname{Re}\left(Z_{j}(u) \mid b_{j} u\right)+\operatorname{Re}\left(u \mid b^{\prime} u\right)_{0},
\end{aligned}
$$

where $b_{j}, b^{\prime} \in \mathcal{C}^{\infty}(M)$. Hence the inequality in (1.13) (with $\bar{Z}$ replacing $Z$ ) follows, with $n=r+2$ and $L_{j}=Z_{j-1}$ for $j=1, \ldots, r+2$.

2.2. The characteristic bundle and the scalar Levi forms. Next we define the characteristic bundle of $\mathfrak{Z}(M)$ and the analogues, for a general distribution $\mathfrak{Z}(M)$, of the scalar Levi forms of $C R$ manifolds.

Definition 2.6. The characteristic bundle of $\mathfrak{Z}(M)$ is the set $H^{0} M \subset T^{*} M$, consisting of the real covectors $\xi$ with $\langle L, \xi\rangle=0$ for all $L \in \mathfrak{Z}(M)$.

If the set $H_{p}^{0} M$ of characteristic covectors at $p \in M$ is $\{0\}$, we say that $\mathfrak{Z}(M)$ is elliptic at $p$.

For each $p \in M$, the set $H_{p}^{0} M=H^{0} M \cap T_{p}^{*} M$ is a vector space. Its dimension $\operatorname{dim}_{\mathbb{R}} H_{p}^{0} M$ is an upper semicontinuous function of $p \in M$. In particular, if $\mathfrak{Z}(M)$ is elliptic at a point $p_{0} \in M$, it is elliptic for $p$ in an open neighborhood $U$ of $p_{0}$. In this case (0.1) is valid with $\epsilon=1$ by Gårding's inequality. Hence obstructions to the validity of the subelliptic estimate (0.1) may come only from the characteristic codirections of $\mathfrak{Z}(M)$.

We restate condition (1.21) in terms of the characteristic bundle.

Proposition 2.7. Condition (1.21) at $p \in M$ is equivalent to

$$
\left\{\begin{array}{c}
\forall \xi \in H_{p}^{0} M, \text { with } \xi \neq 0, \\
\exists Z_{0} \in \mathfrak{Z}(M), Z_{1}, \ldots, Z_{r} \in \mathbb{E}_{\mathfrak{Z}}(M) \cap \overline{\mathbb{E}_{\mathfrak{Z}}(M)}, \\
\text { s.t. } \quad i \xi\left(\left[Z_{1}, \ldots, Z_{r}, \bar{Z}_{0}\right]\right) \neq 0,
\end{array}\right.
$$

Proof. This follows because the elements of $\mathfrak{T}_{\mathfrak{Z}}(M)$ can be expressed as linear combinations of the real parts of the $\left[Z_{1}, \ldots, Z_{r}, \bar{Z}_{0}\right]$ 's, with $Z_{0} \in \mathfrak{Z}(M)$ and $Z_{1}, \ldots, Z_{r} \in \mathbb{E}_{\mathfrak{Z}}(M) \cap \overline{\mathbb{E}_{\mathfrak{Z}}(M)}$, and vice versa, the real and imaginary parts of these $\left[Z_{1}, \ldots, Z_{r}, \bar{Z}_{0}\right]$ belong to $\mathfrak{T}_{\mathfrak{Z}}(M)$.

Definition 2.8. If $\xi \in H_{p}^{0} M$, we define the scalar Levi form of $\mathfrak{Z}(M)$ at $\xi$ as the Hermitian symmetric form

$$
\mathfrak{L}_{\xi}\left(L_{1}, \bar{L}_{2}\right)=i \xi\left(\left[L_{1}, \bar{L}_{2}\right]\right) \quad \text { for } \quad L_{1}, L_{2} \in \mathfrak{Z}(M) .
$$

The value of the right hand side of (2.7) only depends on the values $L_{1}(p)$, $L_{2}(p)$ of the two vector fields $L_{1}, L_{2}$ at the base point $p=\pi(\xi)$. Thus (2.7) is a Hermitian symmetric form on the finite dimensional complex vector space $Z_{p} M$. 
Remark 2.9. In the case where $\mathfrak{Z}(M)$ is the space of $(0,1)$-vector fields of an abstract $C R$ manifold, it was shown in [11] that the subelliptic estimate (0.1) is valid with $\epsilon=1 / 2$ under the assumption that, for every $\xi \in H_{p}^{0} M \backslash\{0\}$, the Levi form $\mathfrak{L}_{\xi}$ is indefinite; this assumption was weakened to allow $\mathfrak{L}_{\xi}=0$ for some nonzero characteristics $\xi$ in [12]. These results suggest that the obstructions to the validity of (0.1) come from the characteristic $\xi$ 's for which $\mathfrak{L}_{\xi} \neq 0$ is semidefinite.

2.3. The distribution $\mathbb{K}_{\mathfrak{Z}}(M)$. Our next aim is to relate $\mathbb{E}_{\mathfrak{Z}}(M)$ and the Levi forms associated to $\mathfrak{Z}(M)$.

Definition 2.10. Define

$$
\begin{gathered}
H^{\oplus} M=\left\{\xi \in H^{0} M \mid \mathcal{L}_{\xi} \geq 0\right\}, \\
\mathbb{K}_{\mathfrak{Z}}(M)=\left\{Z \in \mathfrak{Z}(M) \mid \mathcal{L}_{\xi}(Z, \bar{Z})=0, \forall \xi \in H^{\oplus} M\right\} .
\end{gathered}
$$

Proposition 2.11. For every distribution $\mathfrak{Z}(M) \subset \mathfrak{X}^{\mathbb{C}}(M)$, the set $\mathbb{K}_{\mathfrak{Z}}(M)$ is also a distribution.

Assume in addition that $\mathfrak{Z}(M)$ is formally integrable and that $\mathfrak{Z}(M)$, $\mathfrak{Z}(M) \cap \overline{\mathfrak{Z}(M)}$ are both distributions of constant rank. Then

$$
\mathfrak{Z}(M) \cap \overline{\mathbb{E}_{\mathfrak{Z}}(M)} \subset \mathbb{K}_{\mathfrak{Z}}(M) .
$$

Proof. The first claim is a consequence of the fact that the set of isotropic vectors of a semidefinite Hermitian symmetric form is a complex linear space.

To complete the proof, we need to show that, if $\xi \in H^{\oplus} M$, we have $\mathcal{L}_{\xi}(Z, \bar{Z})=0$ for all $Z \in \mathfrak{Z}(M) \cap \overline{\mathbb{E}_{\mathfrak{Z}}(M)}$. Having fixed $\xi \in H^{\oplus} M$, we can argue in a small coordinate patch $U$ about its base point $p=\pi(\xi)$. By the assumption that $\mathfrak{Z}(M)$ is formally integrable, we can choose real coordinates $x_{1}, \ldots, x_{m}$, centered at $p$, such that, for a pair of nonnegative integers $h, \ell$ with $2 h+\ell \leq m$, setting $z_{j}=x_{j}+i x_{h+j}$ for $j=1, \ldots, h$, a system of generators of $\mathfrak{Z}(M)$ in $U$ is given by the vector fields

$$
\begin{cases}L_{j}=\frac{\partial}{\partial \bar{z}_{j}}+L_{j}^{\prime} & \text { for } j=1, \ldots, h, \\ L_{h+j}=\frac{\partial}{\partial x_{m+1-j}} & \text { for } j=1, \ldots, \ell .\end{cases}
$$

Here $\frac{\partial}{\partial \bar{z}_{j}}=\frac{1}{2}\left(\frac{\partial}{\partial x_{j}}+i \frac{\partial}{\partial x_{h+j}}\right), L_{j}^{\prime} \in \mathfrak{X}^{\mathbb{C}}(U)$ satisfies $L_{j}^{\prime}(0)=0$, and

$$
\left[L_{i}, L_{j}\right]=0 \quad \text { for } \quad 1 \leq i, j \leq h+\ell .
$$

This is obtained by first noticing that the real vector fields in $\mathfrak{Z}(M)$ are a formally integrable distribution of real vector fields. By the classical Frobenius theorem, we can choose a system of local coordinates in which this real distribution is locally generated by the $L_{h+1}, \ldots, L_{h+\ell}$ above. By linear algebra we can obtain, in a neighborhood of $p$, from any basis of $\mathfrak{Z}(M)$ that includes $L_{h+1}, \ldots, L_{h+\ell}$, a new one in which the $L_{1}, \ldots, L_{h}$ have the property that $L_{j}-\frac{\partial}{\partial x_{j}}$ does not contain either $\frac{\partial}{\partial x_{i}}$ for $i=1, \ldots, h$, or $\frac{\partial}{\partial x_{m+1-i}}$ for $i=1, \ldots, \ell$. By this choice we obtain (2.12). Let

$$
L_{j}=\frac{\partial}{\partial \bar{z}_{j}}+\sum_{i=1}^{m-\ell} a_{j}^{i} \frac{\partial}{\partial x_{i}}, \quad \text { with } a_{j}^{i}(0)=0 .
$$


We can assume, by a change of coordinates, that

$$
L_{j}\left(a_{r}^{i}\right)(0)=\frac{\partial a_{r}^{i}(0)}{\partial \bar{z}_{j}}=0 \quad \text { for } \quad j, r=1, \ldots, h, i=2 h+1, \ldots, m-\ell .
$$

In fact, by the formal integrability condition, it follows that

$$
\frac{\partial}{\partial \bar{z}_{j}}+\sum_{i=2 h+1}^{m-\ell}\left(\sum_{r=1}^{h} \frac{\partial a_{j}^{i}(0)}{\partial \bar{z}_{r}} \bar{z}_{r}\right) \frac{\partial}{\partial x_{i}}, \quad \text { for } j=1, \ldots, h,
$$

are commuting vector fields, and, since they also commute with their conjugates, by a change of the coordinates $x_{1}, \ldots, x_{m-\ell}$ we can obtain a new coordinate system for which (2.14) is also satisfied. Let $\xi \in \mathbb{R}^{m}$ be such that $\ell_{j}(0, \xi)=0$ for $j=1, \ldots, h+\ell$, where by $\ell_{j}(x, \xi)$ we indicate the symbols of the differential operators $L_{j}$. This means that the components $\xi_{i}$ of $\xi$ are zero for $1 \leq i \leq 2 h$ and $(m-\ell)<i \leq m$. By the formal integrability condition, there is a second degree homogeneous polynomial $q_{\xi}(x) \in \mathbb{C}\left[x_{1}, \ldots, x_{m-\ell}\right]$ such that

$$
L_{j}\left(i\langle x, \xi\rangle+q_{\xi}(x)\right)=O\left(|x|^{2}\right) \text { for } x \rightarrow 0 .
$$

Next we observe that, by identifying $\xi$ with the corresponding element in $T_{0}^{*} \mathbb{R}^{m}$, and setting $v_{\xi}=\left(i\langle x, \xi\rangle+q_{\xi}(x)\right)$, we obtain

$$
\begin{array}{r}
0=d^{2}\left(i\langle x, \xi\rangle+q_{\xi}(x)\right)(Z, \bar{Z})=Z \bar{Z}\left(v_{\xi}\right)(0)-\bar{Z} Z\left(v_{\xi}\right)(0)-\mathcal{L}_{\xi}(Z, \bar{Z}), \\
\forall Z \in \mathfrak{X}^{\mathbb{C}}(U) .
\end{array}
$$

Thus, in particular, $Z \bar{Z} v_{\xi}(0)=\mathcal{L}_{\xi}(Z, \bar{Z}) \geq 0$ if $Z \in \mathfrak{Z}(M)$ and $\xi \in H_{0}^{\oplus} M$. By (2.14), we have $Z \bar{Z} v_{\xi}(0)=Z \bar{Z} q_{\xi}(0)$. Consider the expression for $q_{\xi}$ as a polynomial in $z_{1}, \ldots, z_{h}, \bar{z}_{1}, \ldots, \bar{z}_{h}, x_{2 h+1}, \ldots, x_{m-\ell}$,

$$
\begin{array}{r}
q_{\xi}(x)=q_{\xi}^{2,0,0}(z, z)+q_{\xi}^{1,1,0}(z, \bar{z})+q_{\xi}^{0,2,0}(\bar{z}, \bar{z})+q_{\xi}^{1,0,1}\left(z, x^{\prime \prime}\right) \\
+q_{\xi}^{0,1,1}\left(\bar{z}, x^{\prime \prime}\right)+q_{\xi}^{0,0,2}\left(x^{\prime \prime}, x^{\prime \prime}\right),
\end{array}
$$

where $x^{\prime \prime}=\left(x_{2 h+1}, \ldots, x_{m-\ell}\right)$. The assumption that $\mathcal{L}_{\xi} \geq 0$ means that $q_{\xi}^{(1,1,0)}(z, \bar{z}) \geq 0$. We can add to $q_{\xi}$ any second degree homogeneous polynomial $f$ in $\mathbb{C}\left[z, x^{\prime \prime}\right]$, since $L_{j}(f)=O\left(|x|^{2}\right)$ for any such polynomial. In this way, we obtain a new $q_{\xi}$, still satisfying (2.16), with the property that

$$
\operatorname{Re}\left(q_{\xi}\right)(x) \geq 0, \forall x \in \mathbb{R}^{m} .
$$

Fix any real valued function $\chi \in \mathcal{C}_{0}^{\infty}\left(\mathbb{R}^{m}\right)$ with $\chi(x)=1$ for $|x| \leq 1,0 \leq$ $\chi(x) \leq 1$ in $\mathbb{R}^{m}$, and $\chi(x)=0$ for $|x| \geq 2$. For large $\tau>0$ the function

$$
u_{\tau}=\sqrt[6]{\tau^{m}} \chi(x \sqrt[3]{\tau}) e^{-\tau\left(i\langle x, \xi\rangle+q_{\xi}(x)\right)}
$$

belongs to $\mathcal{C}_{0}^{\infty}(U)$. We have

$$
\left\|u_{\tau}\right\|_{0}^{2}=\int_{\mathbb{R}^{m}} e^{-2 \sqrt[3]{\tau} q_{\xi}(x)} \chi^{2}(x) d x \leq \int_{\mathbb{R}^{m}} \chi^{2}(x) d x,
$$

because of (2.18). If $Z \in \mathfrak{Z}(M)$, we have:

$$
\begin{aligned}
\left|Z\left(u_{\tau}\right)\right|^{2} & =\sqrt[3]{\tau^{m}}\left|-\tau \chi(x \sqrt[3]{\tau} / 2) Z\left(v_{\xi}\right)+\sqrt[3]{\tau}[Z(\chi)](x \sqrt[3]{\tau})\right|^{2} e^{-2 \tau \operatorname{Re} q_{\xi}(x)} \\
& \leq C_{0} \sqrt[3]{\tau^{m}} \cdot \tau^{2}|x|^{4} \chi(x \sqrt[3]{\tau} / 2) \quad \text { for } \tau \gg 1,
\end{aligned}
$$


with a positive constant $C_{0}$. Indeed $\chi(x \sqrt[3]{\tau} / 2)=1$ when $Z\left(u_{\tau}\right) \neq 0$, and we used the fact that $Z\left(v_{\xi}\right)=O\left(|x|^{2}\right)$ and that $Z(\chi)=O\left(|x|^{2}\right)$. Computing $\left\|Z\left(u_{\tau}\right)\right\|_{0}^{2}$ by making the change of coordinates $y=x \sqrt[3]{\tau}$ shows that, with a constant $C_{1}>0$ independent of $\tau$,

$$
\left\|Z\left(u_{\tau}\right)\right\|_{0}^{2} \leq C_{1} \sqrt[3]{\tau^{2}} \text { for } \tau \gg 1 .
$$

On the other hand, for $u \in \mathcal{C}_{0}^{\infty}(U)$, we have, with a constant $C_{2}>0$ independent of $u$,

$$
\|\bar{Z}(u)\|_{0}^{2} \geq\|Z(u)\|_{0}^{2}-\operatorname{Re}([Z, \bar{Z}](u) \mid u)_{0}-C_{2}\|u\|_{0}^{2} .
$$

For $u=u_{\tau}$ and by using, while computing the integral, the change of variables $y=x \sqrt{\tau}$, we obtain

$$
\begin{aligned}
&-\operatorname{Re}\left([Z, \bar{Z}]\left(u_{\tau}\right) \mid u_{\tau}\right)_{0}=\tau \int \mathcal{L}_{\xi}(Z, \bar{Z}) e^{-2 \operatorname{Re} q_{\xi}(y)} \chi^{2}(y / \sqrt[6]{\tau}) d y \\
&+O\left(\sqrt[3]{\tau^{2}}\right) \quad \text { for } \tau \gg 1 .
\end{aligned}
$$

Since $\chi^{2}(y / \sqrt[6]{\tau})$ is increasing with $\tau$, we get, with a constant $C_{3} \geq 0$,

$$
\begin{aligned}
-\operatorname{Re}\left([Z, \bar{Z}]\left(u_{\tau}\right) \mid u_{\tau}\right)_{0} \geq \tau \int \mathcal{L}_{\xi}(Z, \bar{Z}) e^{-2 \operatorname{Re} q_{\xi}(y)} \chi^{2}(y) d y-C_{3}|\tau|^{\frac{3}{2}} \\
\text { for } \tau \gg 1 .
\end{aligned}
$$

By (2.20) and (2.21), $\left(\sum_{j=1}^{h+\ell}\left\|L_{j}\left(u_{\tau}\right)\right\|_{0}^{2}+\|u\|_{0}^{2}\right)$ is $O\left(\sqrt[3]{\tau^{2}}\right)$ for $\tau \rightarrow \infty$. Thus, by (2.22) and (2.24) we obtain that $\mathcal{L}_{\xi}(Z, \bar{Z})=0$ for $Z \in \mathfrak{Z}(M) \cap \overline{\mathbb{E}_{\mathfrak{Z}}(M)}$.

Remark 2.12. By a slight variant of the proof of Proposition 2.11 we obtain: Assume that $\mathfrak{Z}(M)$ is formally integrable and that $\mathfrak{Z}(M)$ and $\mathfrak{Z}(M) \cap$ $\overline{\mathfrak{Z}(M)}$ are both distributions of constant rank. Let $p \in M$. If there exists $\xi \in H_{p}^{0} M$ such that $\mathcal{L}_{\xi}$ is definite on a complement of $Z_{p} M \cap \overline{Z_{p} M}$ in $Z_{p} M$, then $\mathfrak{Z}(M)$ is not subelliptic at $p$.

Proposition 2.13. For every distribution of complex vector fields $\mathfrak{Z}(M)$, we have

$$
\Theta_{\mathfrak{Z}}(M) \subset \mathbb{K}_{\mathfrak{Z}}(M)
$$

Assume that

$$
\begin{aligned}
\mu_{0}(p) & =\operatorname{dim}_{\mathbb{R}}\left(Z_{p} M+\overline{Z_{p} M}\right) \\
\delta_{0}(p) & =\operatorname{dim}_{\mathbb{R}}\left\langle H_{p}^{\oplus} M\right\rangle \\
\nu_{0}(p) & =\operatorname{dim}_{\mathbb{C}}\left\{Z(p) \in Z_{p} M \mid Z \in \mathbb{K}_{\mathfrak{Z}}(M)\right\}
\end{aligned}
$$

are constant in $M$. Then

$$
\Theta_{\mathfrak{Z}}(M)=\mathbb{K}_{\mathfrak{Z}}(M) .
$$

Finally, if $\mathfrak{Z}(M)$ is formally integrable and of constant rank,

$$
\begin{aligned}
& \mathbb{E}_{\mathfrak{Z}}(M)=\mathfrak{Z}(M)+\overline{\mathbb{K}_{\mathfrak{Z}}(M)}, \\
& \mathbb{A}_{\mathfrak{Z}}(M)=\left\{Z+\bar{Z} \mid Z \in \mathbb{K}_{\mathfrak{Z}}(M)\right\} .
\end{aligned}
$$


Proof. By the definition of $\Theta_{\mathfrak{Z}}(M)$, we have $\mathcal{L}_{\xi}(Z, \bar{Z})=0$ for all $\xi \in H^{\oplus} M$ and $Z \in \Theta_{\mathfrak{Z}}(M)$. Thus (2.25) is always valid.

To prove that, under the additional assumptions, we have the equality (2.29), we apply an argument similar to the one employed in [12, Theorem 2.5].

By the constancy of $\mu_{0}(p)$, the characteristic set $H^{0} M$ is a smooth real vector bundle on $M$. Then the assumption that $\delta_{0}(p)$ is constant implies that $H^{\oplus} M$ generates a smooth linear subbundle of $H^{0} M$, and therefore the quotient $H^{0} M /\left\langle H^{\oplus} M\right\rangle$ is a smooth real linear bundle on $M$.

Since $\nu_{0}(p)$ is constant,

$$
M \ni p \rightarrow K_{p} M=\left\{Z(p) \in Z_{p} M \mid Z \in \mathbb{K}_{\mathfrak{Z}}(M)\right\}
$$

is a complex vector bundle $K M$ on $M$. The map $\left.\xi \rightarrow \mathcal{L}_{\xi}\right|_{K M}$ is injective from the quotient bundle $H^{0} M /\left\langle H^{\oplus} M\right\rangle$ to the bundle Herm $(K M)$ of Hermitian symmetric forms on $K M$. We denote by $L M$ the image bundle.

The dual bundle Herm* $(K M)$ of $\operatorname{Herm}(K M)$ is the real linear subbundle of $K M \otimes_{M} \overline{K M}$ generated by the elements of the form $Z(p) \otimes \bar{Z}(p)$, for $p \in M$ and $Z(p) \in K_{p} M$. The annihilator bundle $L^{0} M$ of $L M$ in $\operatorname{Herm}^{*}(K M)$ contains, for all $p \in M$, positive definite elements of $\operatorname{Herm}^{*}(K M)$. Since the positive definite elements of $L^{0} M$ form an open set in $L^{0} M$, it is easy to construct a global section $\mathfrak{z}$ of $L^{0} M$ with $\mathfrak{z}(p)>0$ for all $p \in M$, by first constructing local sections, and then patching them together by a partition of unity. If $p_{0} \in M$ and $Z \in \mathbb{K}_{3}(M)$, with $Z\left(p_{0}\right) \neq 0$, by the standard Gram-Schmidt orthogonalization process, we can find a smooth function $\phi \in \mathcal{E}(M)$ with $\phi\left(p_{0}\right) \neq 0$ and sections $Z_{2}, \ldots, Z_{k} \in \mathbb{K}_{\mathfrak{Z}}(M)$, such that, by setting $Z_{1}=\phi \cdot Z$, we have $\mathfrak{z}(p)=\sum_{j=1}^{k} Z_{j}(p) \otimes \bar{Z}_{j}(p)$ for $p$ in an open neighborhood $U$ of $p_{0}$ in $M$, where $k$ is the rank of the complex bundle $K M$. The equality

$$
\sum_{j=1}^{k} \mathcal{L}_{\xi}\left(Z_{j}, \bar{Z}_{j}\right)=0 \quad \forall p \in U, \forall \xi \in H_{p}^{0} M
$$

implies that

$$
\sum_{j=1}^{k}\left[Z_{j}, \bar{Z}_{j}\right] \in \mathfrak{Z}(U)+\overline{\mathfrak{Z}(U)} .
$$

By repeating this argument for a set of elements of $\mathbb{K}_{\mathfrak{Z}}(M)$ whose values at $p_{0}$ give a basis for $K_{p_{0}} M$, we prove that every $Z \in \mathbb{K}_{\mathfrak{Z}}(M)$ coincides, on an open neighborhood of $p_{0}$ in $M$, with the restriction of an element of $\Theta_{\mathfrak{Z}}(M)$. Moreover, the number of elements of $\Theta_{\mathfrak{3}}(M)$ that are needed in (2.3) does not exceed $\nu=k^{2}-k$. Thus, if $Z \in \mathbb{K}_{\mathfrak{Z}}(M)$, we can find an open covering $\left\{U_{a}\right\}$ of $M$ and, for each $a$, some $Z_{2}^{(a)}, \ldots, Z_{\nu}^{(a)} \in \Theta_{\mathfrak{Z}}(M)$, for which

$$
[Z, \bar{Z}]+\sum_{j=1}^{\nu}\left[Z_{j}^{(a)}, \bar{Z}_{j}^{(a)}\right] \in \mathfrak{Z}\left(U_{a}\right)+\overline{\mathfrak{Z}\left(U_{a}\right)}, \quad \forall a .
$$

We can assume that the covering $\left\{U_{a}\right\}$ has a finite index. Then, by using a partition of unity $\left\{\chi_{a}\right\}$ subordinated to $\left\{U_{a}\right\}$, and summing together vector fields $\chi_{a} \cdot Z_{j}^{(a)}$ with disjoint supports, we end up with a finite subset 
$Z_{1}, \ldots, Z_{r}$ of vector fields in $\mathfrak{Z}(M)$ such that

$$
\mathcal{L}_{\xi}(Z, \bar{Z})+\sum_{j=1}^{r} \mathcal{L}_{\xi}\left(Z_{j}, \bar{Z}_{j}\right)=0,
$$

showing that $Z \in \Theta_{\mathfrak{Z}}(M)$.

When moreover $\mathfrak{Z}(M)$ is formally integrable and has constant rank, we obtain (2.30) as a consequence of Lemma 2.5 and Proposition 2.11.

Definition 2.14. We say that a higher Levi form concavity condition is satisfied at the point $p \in M$ if

$$
\left\{\begin{array}{c}
\forall \xi \in H_{p}^{0} M \backslash\{0\} \text { with } \mathcal{L}_{\xi} \geq 0, \\
\exists Z_{0} \in \mathfrak{Z}(M), Z_{1}, \ldots, Z_{r} \in \mathbb{K}_{\mathfrak{Z}}(M)+\overline{\mathbb{K}_{\mathfrak{Z}}(M)} \\
\text { with } i \xi\left(\left[Z_{1}, \ldots, Z_{r}, \bar{Z}_{0}\right]\right) \neq 0 .
\end{array}\right.
$$

Definition 2.15. We say that the distribution $\mathfrak{Z}(M)$ is regular at a point $p_{0} \in M$ if its rank, and the functions $\mu_{0}(p)$ of (2.26),$\delta_{0}(p)$ of (2.27), $\nu_{0}(p)$ of (2.28), are all constant in an open neighborhood $U$ of $p_{0}$.

Since the rank of $\mathfrak{Z}(M)$, and the functions $\mu_{0}$ and $\nu_{0}$ are all integral valued and semicontinuous, and $\delta_{0}$ is semicontinuous on the dense open subset where $\mu_{0}$ is constant, the set of regular points of $\mathfrak{Z}(M)$ is open and dense in $M$.

Proposition 2.16. Let $p_{0}$ be a regular point for $\mathfrak{Z}(M)$. Then (2.32) implies (1.21). If in addition we assume that $\mathfrak{Z}(M)$ is formally integrable, then the two conditions (2.32) and (1.21) are equivalent.

Proof. The regularity assumption implies that all $\xi \in H_{p_{0}}^{0} M$ with $\operatorname{ker} \mathcal{L}_{\xi} \supset$ $\Theta_{\mathfrak{Z}}(M)$ belong to the linear span $\left\langle H_{p_{0}}^{\oplus} M\right\rangle$. Thus condition (2.6) holds if $\xi \in H_{p_{0}}^{0} M \backslash\{0\}$ and ker $\mathcal{L}_{\xi} \supset \Theta_{\mathfrak{Z}}(M)$. When ker $\mathcal{L}_{\xi} \not \supset \Theta_{\mathfrak{Z}}(M)$, the restriction of $\mathcal{L}_{\xi}$ to $\Theta_{\mathfrak{Z}}(M)$ is semidefinite, and hence there are $Z_{1}, Z_{2} \in \Theta_{\mathfrak{Z}}(M)$ with $\mathcal{L}_{\xi}\left(Z_{1}, \bar{Z}_{2}\right)=i \xi\left(\left[Z_{1}, \bar{Z}_{2}\right]\right) \neq 0$.

Then because of Propositions 2.7 and 2.13, and Lemma 2.5, we obtain that (2.32) implies condition (1.21) at regular points of $\mathfrak{Z}(M)$.

If in addition $\mathfrak{Z}(M)$ is formally integrable, then by the equality (2.30), the opposite implication is also true.

\section{Pullbacks of Distributions}

Let $M, N$ be smooth real manifolds, and $N \stackrel{\varpi}{\longrightarrow} M$ a smooth submersion.

Given a distribution of complex vector fields $\mathfrak{Z}(M)$, its pullback $\left[\varpi^{*} \mathfrak{Z}\right](N)$ consists of all $W \in \mathfrak{X}^{\mathbb{C}}(N)$ with the property that

(3.1) $\forall U^{\text {open }} \subset M, \forall \sigma \in \mathcal{C}^{\infty}(U, N)$, with $\varpi \circ \sigma=\operatorname{id}_{U}, d \varpi^{\mathbb{C}}(W \circ \sigma) \in \mathfrak{Z}(U)$.

Let

$$
\begin{aligned}
V^{\varpi} N & =\{v \in T N \mid d \varpi(v)=0\} & & \text { be the vertical bundle, and } \\
\mathfrak{V}^{\varpi}(N) & =\mathcal{C}^{\infty}\left(N, V^{\varpi} N\right) & & \text { the vertical distribution. }
\end{aligned}
$$

Lemma 3.1. Let $N \stackrel{\varpi}{\longrightarrow} M$ be a smooth submersion. Then

$$
\mathfrak{V}^{\varpi}(N) \subset\left[\varpi^{*} \mathfrak{Z}\right](M) \quad \text { and } \quad\left[\mathfrak{V}^{\varpi}(N),\left[\varpi^{*} \mathfrak{Z}\right](N)\right] \subset\left[\varpi^{*} \mathfrak{Z}\right](N) .
$$


Proof. We note that $\left[\varpi^{*} \mathfrak{Z}\right](N)$ is the space of global sections of a fine sheaf of left $\mathcal{C}^{\infty}$-modules. Thus by localization we can reduce the discussion to the case where $N=M \times \Omega$ for an open subset $\Omega$ of a Euclidean space $\mathbb{R}^{k}$, and $\varpi$ is the projection onto the first factor, in which situation the statement is trivial.

Definition 3.2. If $N \stackrel{\iota}{\rightarrow} M$ is a smooth immersion, we define the pullback $\left[\iota^{*} \mathfrak{Z}\right](N)$ of $\mathfrak{Z}(M)$ to $N$ to be the set of complex vector fields $Z^{\prime} \in \mathfrak{X}^{\mathbb{C}}(N)$ having the following property

$$
\left\{\begin{array}{c}
\forall q_{0} \in N, \exists V^{\text {open }} \subset N \text { with } V \ni p_{0}, \text { and } Z \in \mathfrak{Z}(M) \\
\text { s.t. } \quad d \iota(q)\left(Z^{\prime}(q)\right)=Z(\iota(q)) \forall q \in V .
\end{array}\right.
$$

Let $M, N$ be smooth manifolds. A smooth map $N \stackrel{\phi}{\rightarrow} M$ is a submersion onto its image if there exists a smooth manifold $S$, a submersion $N \stackrel{\varpi}{\longrightarrow} S$ and an immersion $S \stackrel{\iota}{\rightarrow} M$ that factorize $\phi$, i.e. that make the following diagram commute:

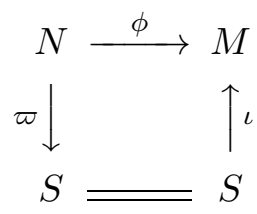

Definition 3.3. The pullback of $\mathfrak{Z}(M)$ by a map $N \stackrel{\phi}{\rightarrow} M$, which is a submersion onto its image, is the distribution of complex vector fields

$$
\left[\phi^{*} \mathfrak{Z}\right](N)=\left[\varpi^{*}\left[\iota^{*} \mathfrak{Z}\right]\right](N),
$$

where $\varpi$ and $\iota$ are the maps in (3.6) .

Remark 3.4. If $N$ is an open subset of $M$ and $\iota: N \hookrightarrow M$ is the inclusion, then $\iota^{*}(\mathfrak{Z})(N)=\mathfrak{Z}(N)$ is the distribution on $N$ that is generated by the restrictions to $N$ of the vector fields $Z \in \mathfrak{Z}(M)$.

More generally, if $N \subset M$ is a smooth submanifold, and $\iota: N \hookrightarrow M$ the embedding map, then $\left[\iota^{*} \mathfrak{Z}\right](N)$ is the distribution generated by the restrictions to $N$ of the vector fields $Z \in \mathfrak{Z}(M)$ with $Z(p) \in T_{p}^{\mathbb{C}} N$ for every $p \in N$.

Definition 3.5. Let $M, N$ be smooth manifolds, and $\mathfrak{Z}_{M}(M), \mathfrak{Z}_{N}(N)$ distributions of complex vector fields on $M$ and $N$, respectively. Let $N \stackrel{\phi}{\rightarrow} M$ be a submersion onto its image. We say that $\phi$ is a $Z$-morphism if

$$
\mathfrak{Z}_{N}(N) \subset\left[\phi^{*} \mathfrak{Z}_{M}\right](M) .
$$

Remark 3.6. We keep the notation of Definition 3.5, and let $\varpi, \iota$ be the maps in (3.6). Set $\mathfrak{Z}_{S}(S)=\left[\iota^{*} \mathfrak{Z}_{M}\right](S)$. Then $N \stackrel{\phi}{\rightarrow} M$ is a $Z$-morphism if and only if $N \stackrel{\varpi}{\longrightarrow} S$ is a $Z$-morphism.

Lemma 3.7. Let $M, N$ be two smooth real manifolds, and $N \stackrel{\varpi}{\rightarrow} M$ be a smooth submersion. Then

$$
\begin{aligned}
\mathbb{E}_{\varpi^{*} \mathfrak{Z}_{M}}(N) & =\left[\varpi^{*} \mathbb{E}_{\mathfrak{Z}_{M}}\right](N) \\
\mathbb{K}_{\varpi^{*} \mathfrak{Z}_{M}}(N) & =\left[\varpi^{*} \mathbb{K}_{\mathfrak{Z}_{M}}\right](N) \\
\Theta_{\varpi^{*} \mathfrak{Z}_{M}}(N) & =\left[\varpi^{*} \Theta_{\mathfrak{Z}_{M}}\right](N) .
\end{aligned}
$$


Proof. Again the statement becomes trivial after, by localization, we reduce to the case where $N=M \times \Omega$ with $\Omega$ open in $\mathbb{R}^{k}$ and $\varpi$ being the projection onto the first factor.

Proposition 3.8. Let $M$ and $N$ be smooth real manifolds, with assigned complex valued distributions of smooth complex vector fields $\mathfrak{Z}_{M}(M)$ and $\mathfrak{Z}_{N}(N)$, respectively. Let $N \stackrel{\phi}{\rightarrow} M$ be a smooth submersion onto the image and $a Z$-morphism. Let $q_{0} \in N$ and $p_{0}=\phi\left(q_{0}\right)$ be a regular point of $\mathfrak{Z}_{M}(M)$, according to Definition 2.15, Assume that

(1) $\mathfrak{Z}_{N}(N)$ satisfies the higher Levi form concavity condition (2.32) at the point $q_{0}$;

(2) the pullback $\phi_{q_{0}}^{*}: H_{p_{0}}^{0} M \rightarrow H_{q_{0}}^{0} N$ is injective.

Then conditions (2.32) and (1.21) at $p_{0}$ are valid for $\mathfrak{Z}_{M}(M)$.

Proof. Using Remark 3.6 we shall split the proof by separately considering the case in which $\phi$ is a submersion and the case where $\phi$ is an immersion.

First we assume that $N \stackrel{\phi}{\rightarrow} M$ is a smooth submersion. In this case $\mathbb{E}_{\mathfrak{Z}_{N}}(N) \subset \mathbb{E}_{\phi^{*} \mathfrak{Z}_{M}}(N)$. Moreover, (2) is automatically satisfied because $\mathfrak{Z}_{N}(N) \subset\left[\phi^{*} \mathfrak{Z}_{M}\right](N)$. The statement is trivially true, as it is easily checked by reducing it to the case where $N=M \times \Omega$, with $\Omega$ open in $\mathbb{R}^{k}$ and $\varpi$ the projection onto the first coordinate.

To complete the proof of the general case, it suffices, by localization about $q_{0}$, to consider the case in which $N=S$ is a smooth submanifold of $M$, and $\mathfrak{Z}_{N}(N)$ are the restrictions to $N$ of elements of $\mathfrak{Z}_{M}(M)$ with real and imaginary parts tangent to $N$ at all points of $N$. By (2), (2.32) for $\mathfrak{Z}_{N}(N)$ at $q_{0}=p_{0}$, implies that (2.32) is satisfied at $p_{0}$ for $\mathfrak{Z}_{M}(M)$. By the regularity assumption, this implies (1.21) at $p_{0}$ for $\mathfrak{Z}_{M}(M)$.

\section{Hypoellipticity for some Differential operators of the FIRST AND OF THE SECOND ORDER}

We keep the notation of $₫ 1$, $₫ 2$. Theorems 4.1, Corollary 4.2, and Theorem 4.3 below, which concern systems of first order partial differential operators, and second order partial differential operators closely related to sums of squares of vector fields, directly follow from the assumption that $\mathfrak{Z}(M)$ be subelliptic. The other results of this section, namely Theorems 4.4 and 4.8 , refer to generalized parabolic second order operators, and are proved under conditions (4.26) and (4.43), respectively, that more directly involve the Lie structure of $\mathfrak{Z}(M)$ with respect to some generalized time vector field.

Let $E \stackrel{\pi}{\rightarrow} M$ be a complex vector bundle of rank $r$ on $M$, endowed with a $\mathbb{C}$-linear connection

$$
\nabla: \mathfrak{X}(M) \times \mathcal{C}^{\infty}(M, E) \rightarrow \mathcal{C}^{\infty}(M, E) .
$$

In a local trivialization $\left.E\right|_{U} \simeq U \times \mathbb{C}^{r}$, the connection $\nabla$ is described by the datum of a $\mathfrak{g l}(r, \mathbb{C})$-valued smooth one form $\gamma=\left(\gamma_{\beta}^{\alpha}\right) \in \mathcal{C}^{\infty}(U, \mathfrak{g l}(r, \mathbb{C}) \otimes$ $\left.T^{*} M\right)$. Using upper Greek letters for the components of the sections in $\mathcal{C}^{\infty}(U, E) \simeq \mathcal{C}^{\infty}\left(U, \mathbb{C}^{r}\right)$, we have

$$
\left(\nabla_{X}(\sigma)\right)^{\alpha}=X \sigma^{\alpha}+\sum_{\beta=1}^{r} \gamma_{\beta}^{\alpha}(X) \sigma^{\beta}, \quad \text { for } \alpha=1, \ldots, r .
$$


By $\mathbb{C}$-linearity, we can define, for each complex valued vector field $Z \in \mathfrak{X}^{\mathbb{C}}$, a linear partial differential operator

$$
\nabla_{Z}: \mathcal{C}^{\infty}(M, E) \ni \sigma \rightarrow \nabla_{\operatorname{Re} Z}(\sigma)+i \nabla_{\operatorname{Im} Z}(\sigma) \in \mathcal{C}^{\infty}(M, E) .
$$

Let us fix a smooth Riemannian metric $g$ on $M$ and a smooth Hermitian metric $h$ on the fibers of $E \stackrel{\pi}{\rightarrow} M$. Then we can define the formal adjoint $\nabla_{Z}^{*}: \mathcal{C}^{\infty}(M, E) \rightarrow \mathcal{C}^{\infty}(M, E)$ by

$$
\begin{gathered}
\int_{M} h\left(\nabla_{Z}^{*} u, v\right) d \lambda_{g}=\int_{M} h\left(u, \nabla_{Z} v\right) d \lambda_{g} \\
\forall u, v \in \mathcal{C}^{\infty}(M, E), \text { with } \operatorname{supp}(u) \cap \operatorname{supp}(v) \Subset M,
\end{gathered}
$$

where $d \lambda_{g}$ is the Lebesgue density on $M$ with respect to the Riemannian metric $g$.

We obtain an analogue of [12, Theorem 4.1]:

Theorem 4.1. Let $E \stackrel{\pi}{\rightarrow} M$ be a smooth complex vector bundle of rank $r$ on $M$ and $\nabla$ a $\mathbb{C}$-linear connection on $E \stackrel{\pi}{\rightarrow} M$. If $\mathfrak{Z}(M)$ is subelliptic at a point $p_{0} \in M$, then any weak solution $u \in L_{\mathrm{loc}}^{2}(M, E)$ of

$$
\nabla_{Z} u \in \mathcal{C}^{\infty}(M, E) \quad \forall Z \in \mathfrak{Z}(M)
$$

is equal, a.e. in an open neighborhood $U$ of $p_{0}$ in $M$, to a smooth section of $E \stackrel{\pi}{\rightarrow} M$.

Formula (4.5) means that for every $Z \in \mathfrak{Z}(M)$ there is a smooth section $f_{Z} \in \mathcal{C}^{\infty}(M, E)$ such that

$$
\begin{aligned}
\int_{M} h\left(u, \nabla_{Z}^{*} v\right) d \lambda_{g}=\int_{M} h\left(f_{Z}, v\right) d \lambda_{g}, \\
\quad \forall v \in \mathcal{C}^{\infty}(M, E), \text { with } \operatorname{supp}(v) \Subset M .
\end{aligned}
$$

Proof. The statement is local. Therefore by substituting for $M$ a relatively compact open neighborhood of $p_{0}$, we can assume that $\mathfrak{Z}(M)$ is generated by a finite set of vector fields $L_{1}, \ldots, L_{n}$, and that, for some $\epsilon>0$ and $C>0$, we have the estimate

$$
\|v\|_{\epsilon}^{2} \leq C\left(\sum_{j=1}^{n}\left\|L_{j}(v)\right\|_{0}^{2}+\|v\|_{0}^{2}\right), \quad \forall v \in \mathcal{C}_{0}^{\infty}(M) .
$$

We can also assume that $E$ is the trivial bundle $M \times \mathbb{C}^{r}$ on $M$, so that (4.5) is equivalent to the system

$$
L_{j} u^{\alpha}+\sum_{\beta=1}^{r} a_{j \beta}^{\alpha}(p) u^{\beta}=f_{j}^{\alpha} \in \mathcal{C}^{\infty}(M), \quad \text { for } j=1, \ldots, n, \alpha=1, \ldots, r,
$$

with $a_{j \beta}^{\alpha} \in \mathcal{C}^{\infty}(M)$. Defining

$$
\left\{\begin{aligned}
& \mathfrak{d}: \mathcal{C}^{\infty}\left(M, \mathbb{C}^{r}\right) \rightarrow\left[\mathcal{C}^{\infty}\left(M, \mathbb{C}^{r}\right)\right]^{n} \quad \text { by } \\
& \mathfrak{d}\left(\left(u^{\alpha}\right)_{\alpha=1, \ldots, r}\right)=\left(L_{j} u^{\alpha}+\sum_{\beta=1}^{r} a_{j \beta}^{\alpha}(p) u^{\beta}\right)_{\substack{1 \leq \alpha \leq r \\
1 \leq j \leq n}}
\end{aligned}\right.
$$


we obtain from (4.7) that, with some new constant $C>0$ and the same $\epsilon>0$ :

$$
\|v\|_{\epsilon}^{2} \leq C\left(\|\mathfrak{d}(v)\|_{0}^{2}+\|v\|_{0}^{2}\right), \quad \forall v \in \mathcal{C}_{0}^{\infty}\left(M, \mathbb{C}^{r}\right) .
$$

We have, for $\chi \in \mathcal{C}_{0}^{\infty}(M)$,

$$
(\mathfrak{d}(\chi u))_{j}^{\alpha}=\chi(\mathfrak{d}(u))_{j}^{\alpha}+\left(L_{j}(\chi) u^{\alpha}\right) .
$$

In particular, if $u \in L_{\text {loc }}^{2}\left(M, \mathbb{C}^{r}\right)$ is a weak solution of (4.8), we have that $\mathfrak{d}(\chi u) \in\left[L^{2}\left(M, \mathbb{C}^{r}\right)\right]^{n}$. By applying Friedrichs' theorem on the identity of the weak and strong extensions of a first order partial differential operator, we can find a sequence $\left\{v_{\nu}\right\} \subset \mathcal{C}_{0}^{\infty}\left(M, \mathbb{C}^{r}\right)$ such that

$$
v_{\nu} \rightarrow \chi u \text { in } L^{2}\left(M, \mathbb{C}^{r}\right) \text { and } \mathfrak{d}\left(v_{\nu}\right) \rightarrow \mathfrak{d}(\chi u) \text { in }\left[L^{2}\left(M, \mathbb{C}^{r}\right)\right]^{n} .
$$

The subelliptic estimate (4.10) yields a uniform bound for the Sobolev $\epsilon$ norm $\left\|v_{\nu}\right\|_{\epsilon}$. This implies that $\chi u \in W^{\epsilon}\left(M, \mathbb{C}^{r}\right)$, where $W^{\epsilon}\left(M, \mathbb{C}^{r}\right)$ denotes the Sobolev space of $L^{2}$-vector valued functions that have $L^{2}$-derivatives of the positive real order $\epsilon$. Hence $u \in W_{\mathrm{loc}}^{\epsilon}\left(M, \mathbb{C}^{r}\right)$.

To show that $u \in \mathcal{C}^{\infty}\left(M, \mathbb{C}^{r}\right)$, we use the Sobolev embedding theorem: it suffices to show that $u \in W_{\mathrm{loc}}^{s}\left(M, \mathbb{C}^{r}\right)$ for all $s>0$. Assume that we already know that this is true for some $s_{0}>0$. If $\chi \in \mathcal{C}_{0}^{\infty}(M)$, then

$$
\mathfrak{d}(\chi u)=[\mathfrak{d}, \chi](u)+\chi \mathfrak{d}(u) \in W^{s_{0}}\left(M, \mathbb{C}^{r}\right)
$$

and has compact support. Fix any scalar pseudodifferential operator $\Lambda_{q} \in$ $\Psi^{q}(M)$, with $s_{0}-\epsilon<q \leq s_{0}$. Then, by the continuity properties of classical pseudodifferential operators, we obtain

$$
\mathfrak{d}\left(\Lambda_{q}(\chi u)\right)=\left[\mathfrak{d}, \Lambda_{q}\right](\chi u)+\Lambda_{q}(\mathfrak{d}(\chi u)) \in L_{\mathrm{loc}}^{2}\left(M, \mathbb{C}^{r}\right),
$$

because $\Lambda_{q}$ and the commutator $\left[\mathfrak{d}, \Lambda_{q}\right](u)$ have orders $\leq s_{0}$ and $\chi u, \mathfrak{d}(\chi u) \in$ $W^{s_{0}}(M)$ and have compact support in $M$. Thus, by the argument above, $\Lambda_{q}(\chi u) \in W^{\epsilon}\left(M, \mathbb{C}^{r}\right)$. This implies that $\chi u \in W^{q+\epsilon}\left(M, \mathbb{C}^{r}\right)$, with $q+\epsilon>s$. Since $\chi$ was an arbitrary smooth function with compact support in $M$, this yields $u \in W_{\mathrm{loc}}^{q+\epsilon}\left(M, \mathbb{C}^{r}\right)$. By recurrence we obtain that $u \in W_{\mathrm{loc}}^{s}\left(M, \mathbb{C}^{r}\right)$ for all $s>0$, and hence is equal a.e. to a smooth section. The proof is complete.

Corollary 4.2. Assume that $M$ is compact and that $\mathfrak{Z}(M)$ is subelliptic at all points $p \in M$. Then

(1) the space

$$
\mathcal{O}_{\mathfrak{Z}}(M)=\left\{\sigma \in \mathcal{C}^{\infty}(M, E) \mid \nabla_{Z}(\sigma)=0, \forall Z \in \mathfrak{Z}(M)\right\}
$$

is finite dimensional.

(2) The map

$$
\mathcal{C}^{\infty}(M, E) \times \mathfrak{Z}(M) \ni(\sigma, Z) \rightarrow\left(\nabla_{Z}(\sigma), Z\right) \in \mathcal{C}^{\infty}(M, E) \times \mathfrak{Z}(M)
$$

has a closed range. By this we mean that, if $\left\{\sigma_{\nu}\right\}$ is a sequence of sections in $\mathcal{C}^{\infty}(M, E)$ and for each $Z \in \mathfrak{Z}(M)$ there is $f_{Z} \in \mathcal{C}^{\infty}(M, E)$ such that $\nabla_{Z}\left(\sigma_{\nu}\right)$ converges uniformly to $f_{Z}$ in $M$, then there exists a section $\sigma \in \mathcal{C}^{\infty}(M, E)$ with $\nabla_{Z}(\sigma)=f_{Z}$ for all $Z \in \mathfrak{Z}(M)$. 
Proof. (1) follows from the Sobolev embedding theorem, because $\mathcal{O}_{\mathfrak{Z}}(M)$ is an $L^{2}$-closed subspace of $\mathcal{C}^{\infty}(M, E)$ on which the $L^{2}$ and the $\epsilon$-Sobolev norm, for some $\epsilon>0$, are equivalent. Finally, (2) is a consequence of the fact that, since $M$ is compact, for a finite set $L_{1}, \ldots, L_{n} \in \mathfrak{Z}(M)$, some $\epsilon>0$ and some const $>0$, on the $L^{2}$-orthogonal complement of $\mathcal{O}_{\mathfrak{Z}}(M)$, we have the coercive estimate

$$
\|u\|_{\epsilon}^{2} \leq \mathrm{const}\left(\sum_{j=1}^{n}\left\|L_{j}(u)\right\|_{0}^{2}\right), \quad \forall u \in \mathcal{C}^{\infty}(M, E) \cap\left[\mathcal{O}_{\mathfrak{Z}}(M)\right]^{\perp} .
$$

Theorem 4.3. Let $\mathfrak{Z}(M)$ be a distribution of complex vector fields.

(1) We can find a locally finite family $\left\{L_{j}\right\} \subset \mathfrak{Z}(M)$, such that, for any choice of $a \in \mathcal{C}^{\infty}(M)$, the second order operator

$$
P(u)=a u+\sum_{j} \bar{L}_{j} L_{j}(u)
$$

is hypoelliptic at all points of $M$ at which $\mathfrak{Z}(M)$ is subelliptic.

(2) If $\mathfrak{Z}(M)$ is finitely generated then, for any set of generators $L_{1}, \ldots, L_{n}$ of $\mathfrak{Z}(M)$, and for any choice of $Z_{0} \in \mathfrak{Z}(M)+\overline{\mathfrak{Z}(M)}$ and $a \in \mathcal{C}^{\infty}(M)$, the operator

$$
P(u)=\sum_{j=1}^{n} \bar{L}_{j} L_{j}(u)+Z_{0}(u)+a u
$$

is hypoelliptic at all points $p$ of $M$ where $\mathfrak{Z}(M)$ is subelliptic.

Let $M^{\prime}$ be the open subset of $M$ of points $p$ where $\mathfrak{Z}(M)$ is subelliptic. Then the operators $P$ of (1) and (2) satisfy the following:

$$
\left\{\begin{array}{c}
\forall U^{\text {open }} \Subset M^{\prime}, \quad \exists \epsilon>0, C>0 \text { such that } \\
\|u\|_{\epsilon}^{2} \leq C\left(\left|(P(u) \mid u)_{0}\right|+\|u\|_{0}^{2}\right), \quad \forall u \in \mathcal{C}_{0}^{\infty}(U) .
\end{array}\right.
$$

Proof. Let $\left\{U_{\nu}\right\}$ be an open covering of $M^{\prime}$ by relatively compact open subsets, and, for each of them, let $L_{1}^{(\nu)}, \ldots, L_{n_{\nu}}^{(\nu)} \in \mathfrak{Z}(M)$ be chosen in such a way that, for suitable $\epsilon_{\nu}>0, C_{\nu}>0$, we have the estimate

$$
\|u\|_{\epsilon_{\nu}}^{2} \leq C_{\nu}\left(\sum_{h=1}^{n_{\nu}}\left\|L_{h}^{(\nu)}(u)\right\|_{0}^{2}+\|u\|_{0}^{2}\right), \quad \forall u \in \mathcal{C}_{0}^{\infty}\left(U_{\nu}\right) .
$$

Take smooth functions $\chi_{\nu} \in \mathcal{C}_{0}^{\infty}\left(U_{\nu}\right)$ such that $\operatorname{supp}\left(\chi_{\nu}\right) \Subset U_{\nu}$, the family $\left\{\operatorname{supp}\left(\chi_{\nu}\right)\right\}$ is locally finite, and $\sum_{\nu}\left|\chi_{\nu}(p)\right|^{2}>0$ for all $p \in M^{\prime}$. Then (1) holds with $\left\{L_{j}\right\}=\left\{\chi_{\nu} L_{h}^{(\nu)}\right\}$.

By [19, the hypoellipticity of (4.17) and of (4.18) is a consequence of (4.20). Since it suffices to prove that for each $p \in M^{\prime}$, there is a small open neighborhood $U \Subset M^{\prime}$ of $p$ for which (4.20) holds true, we can reduce the proof to the case where $\mathfrak{Z}(M)$ is the $\mathcal{C}^{\infty}(M)$-module generated by $L_{1}, \ldots, L_{n} \in \mathfrak{Z}(M)$, and the operator $P$ is of the form (4.18).

By integration by parts we obtain

$$
\begin{array}{r}
-(P u \mid u)_{0}=\sum_{j=1}^{n}\left\|L_{j}(u)\right\|_{0}^{2}-\left(L_{0}(u) \mid u\right)_{0}+\left(u \mid L_{n+1}(u)\right)_{0}+\left(a^{\prime} u \mid u\right)_{0} \\
\forall u \in \mathcal{C}_{0}^{\infty}(M) .
\end{array}
$$


Since $L_{1}, \ldots, L_{n}$ generate $\mathfrak{Z}(M)$, we obtain that $(0.1)$ is valid for every relatively compact open subset $U \Subset M^{\prime}$, and this in turn, together with (4.21), implies (4.20). The proof is complete.

Theorem 4.4. We keep the notation of Definition 1.11. Assume that $\mathfrak{Z}(M)$ is generated by a finite set $L_{1}, \ldots, L_{n}$ of complex vector fields. Let $X_{0} \in$ $\mathfrak{X}(M)$ be a real vector field. Let

$$
\begin{aligned}
\mathbb{T}_{0}^{\prime}(M) & =\mathcal{C}^{\infty}(M, \mathbb{R}) \cdot X_{0}+\mathfrak{T}_{\mathfrak{Z}}(M) \\
\mathbb{T}_{h}^{\prime}(M) & =\left[\mathfrak{A}_{\mathfrak{Z}}(M), \mathbb{T}_{h-1}^{\prime}(M)\right], \quad \text { for } h \geq 1, \\
\mathfrak{T}^{\prime}(M) & =\sum_{h=0}^{\infty} \mathbb{T}_{h}^{\prime}(M) .
\end{aligned}
$$

In particular, $\mathfrak{T}^{\prime}(M)$ is the $\mathfrak{A}_{\mathfrak{Z}}(M)$-Lie-submodule of $\mathfrak{X}(M)$ generated by $X_{0}$ and $\mathfrak{T}_{\mathfrak{Z}}(M)$. Then, for any choice of $Y_{0} \in \mathbb{T}_{\mathfrak{Z}}^{(0)}(M)$ and $a \in \mathcal{C}^{\infty}(M)$, the second order partial differential operator

$$
P(u)=a \cdot u+X_{0}(u)+i Y_{0}(u)+\sum_{j=1}^{n} \bar{L}_{j} L_{j}(u)
$$

is hypoelliptic at all points $p \in M$ where

$$
\left\{X(p) \mid X \in \mathfrak{T}^{\prime}(M)\right\}=T_{p} M .
$$

We divide the proof of Theorem 4.4 into several steps. First we prove

Lemma 4.5. Let $U \Subset M$ be an open set and assume that there are $\epsilon>0$, $C>0$ and $A_{1}, \ldots, A_{r} \in \Psi^{0}(U)$ such that

$$
\left\{\begin{array}{r}
\|u\|_{\epsilon}^{2}+\sum_{j=1}^{n}\left\|L_{j}(u)\right\|_{0}^{2} \leq C\left(\sum_{h=1}^{r}\left|\left(P(u) \mid A_{h}(u)\right)_{0}\right|+\|u\|_{0}^{2}\right), \\
\forall u \in \mathcal{C}_{0}^{\infty}(U) .
\end{array}\right.
$$

Then for every real $s \geq 0$, and every open subset $U^{\prime}$ with $U^{\prime} \Subset U$, there is a constant $C^{\prime}=C\left(s, U^{\prime}\right)$ such that

$$
\|u\|_{s+\epsilon}^{2}+\sum_{j=1}^{n}\left\|L_{j}(u)\right\|_{s}^{2} \leq C^{\prime}\left(\|P(u)\|_{s}^{2}+\|u\|_{0}^{2}\right), \quad \forall u \in \mathcal{C}^{\infty}\left(U^{\prime}\right) .
$$

Proof. Let $\Lambda_{s} \in \Psi^{s}(U)$ be elliptic. Then we have, with real constants $C_{1}>0, C_{2} \geq 0$, uniformly for $u \in \mathcal{C}_{0}^{\infty}\left(U^{\prime}\right)$,

$$
\begin{aligned}
\|u\|_{s+\epsilon}^{2}+\sum_{j=1}^{n}\left\|L_{j}(u)\right\|_{s}^{2} & \leq C_{1}\left(\left\|\Lambda_{s}(u)\right\|_{\epsilon}^{2}+\sum_{j=1}^{n}\left\|\Lambda_{s}\left(L_{j}(u)\right)\right\|_{0}^{2}\right) \\
& \leq C_{1}\left(\left\|\Lambda_{s}(u)\right\|_{\epsilon}^{2}+\sum_{j=1}^{n}\left\|L_{j}\left(\Lambda_{s}(u)\right)\right\|_{0}^{2}\right)+C_{2}\|u\|_{s}^{2}
\end{aligned}
$$

Thus, using the inequality

$$
\left\{\begin{array}{c}
\forall \delta>0, \quad \exists C_{\delta}>0 \quad \text { s.t } \\
\|u\|_{s}^{2} \leq \delta\|u\|_{s+\epsilon}^{2}+C_{\delta}\|u\|_{0}^{2}, \quad \forall u \in \mathcal{C}_{0}^{\infty}(U),
\end{array}\right.
$$


we obtain that the left hand side of (4.28) is bounded by a constant times

$$
\sum_{h=1}^{r}\left|\left(P\left(\Lambda_{s}(u)\right) \mid A_{h}\left(\Lambda_{s}(u)\right)\right)_{0}\right|+\|u\|_{0}^{2} .
$$

The operator $Q=P+\sum_{j=1}^{n} L_{j}^{*} L_{j}$ is an operator of the first order, with principal part $X_{0}+i Y_{0}$. Therefore if $A \in \Psi^{0}(U)$, we obtain

$$
\begin{aligned}
\left(P\left(\Lambda_{s}(u)\right) \mid A\left(\Lambda_{s}(u)\right)_{0}=\right. & \left(\Lambda_{s}(Q(u)) \mid A\left(\Lambda_{s}(u)\right)\right)_{0}+O\left(\|u\|_{s}^{2}\right) \\
& -\sum_{j=1}^{n}\left(L_{j}\left(\Lambda_{s}(u)\right) \mid L_{j}\left(\Lambda_{s}(A(u))\right)\right)_{0} \\
= & \left(\Lambda_{s}(Q(u)) \mid A\left(\Lambda_{s}(u)\right)\right)_{0}+O\left(\|u\|_{s}^{2}\right) \\
& +\sum_{j=1}^{n}\left(\bar{L}_{j} \Lambda_{s} L_{j}(u) \mid A\left(\Lambda_{s}(u)\right)\right)_{0}+O\left(\left\|L_{j}(u)\right\|_{s}\|u\|_{s}\right) \\
+ & \sum_{j=1}^{n}\left(\left[\Lambda_{s}, L_{j}\right](u) \mid\left[L_{j}, A \circ \Lambda_{s}\right](u)\right)_{0} \\
& +\sum_{j=1}^{n}\left(\left[\Lambda_{s}, L_{j}\right](u) \mid A \circ \Lambda_{s}\left(L_{j}(u)\right)\right)_{0} \\
= & \left(\Lambda_{s}(P(u)) \mid A \circ \Lambda_{s}(u)\right)_{0}+O\left(\|u\|_{s}^{2}+\sum_{j=1}^{n}\left\|L_{j}(u)\right\|_{s}^{2}\right), \\
& \forall u \in \mathcal{C}^{\infty}\left(U^{\prime}\right),
\end{aligned}
$$

where we use $O(N(u))$ to indicate some quantity whose modulus is bounded by a constant times $N(u)$. This computation yields

$$
\left\{\begin{array}{r}
\forall U^{\prime} \Subset U, \forall s \in \mathbb{R}_{+}, \exists A_{1}^{(2 s)}, \ldots, A_{r}^{(2 s)} \in \Psi^{(2 s)}(U), \exists C_{s}^{\prime}>0 \text { s.t } \\
\|u\|_{s+\epsilon}^{2}+\sum_{j=1}^{n}\left\|L_{j}(u)\right\|_{s}^{2} \leq C_{s}^{\prime}\left(\sum_{h=1}^{r}\left|\left(P(u) \mid A_{h}^{(2 s)}(u)\right)_{0}\right|+\|u\|_{0}^{2}\right), \\
\forall u \in \mathcal{C}_{0}^{\infty}\left(U^{\prime}\right) .
\end{array}\right.
$$

Clearly (4.29) implies (4.28).

It is known (see e.g. [14, 18]) that

Lemma 4.6. If (4.28) is valid for all $s \in \mathbb{R}_{+}$and all open subset $U^{\prime} \Subset U$, then $P$ is $\mathcal{C}^{\infty}$-hypoelliptic in $U$.

End of the proof of Theorem 4.4. By the previous Lemmas, we only need to prove (4.27). First we note that, for all $u \in \mathcal{C}_{0}^{\infty}(U)$,

$$
\begin{aligned}
\left|\operatorname{Re}\left(X_{0}(u) \mid u\right)_{0}\right| & =\frac{1}{2}\left|\int_{U} X_{0}(u \bar{u}) d \lambda_{g}\right| \\
& =\left.\frac{1}{2}\left|\int_{U}\right| u\right|^{2} X_{0}^{*}(1) d \lambda_{g} \mid \leq \frac{1}{2}\left(\sup _{p \in U}\left|X_{0}^{*}(1)\right|\right)\|u\|_{0}^{2}
\end{aligned}
$$


Since, for some positive constant $C_{0}$ depending on $U$,

$$
\left|\left(i Y_{0}(u)+a u \mid u\right)_{0}\right| \leq C_{0}\|u\|_{0}\left(\|u\|_{0}+\sum_{j=1}^{n}\left\|L_{j}(u)\right\|_{0}\right), \quad \forall u \in \mathcal{C}_{0}^{\infty}(U),
$$

we obtain, upon integrating by parts, that with a constant $C_{1}>0$,

$$
\sum_{j=1}^{n}\left\|L_{j}(u)\right\|_{0}^{2} \leq-2(P(u) \mid u)_{0}+C_{1}\|u\|_{0}^{2}, \quad \forall u \in \mathcal{C}_{0}^{\infty}(U) .
$$

Next we note that, taking for $A_{0} \in \Psi^{0}(U)$ the composition with $X_{0}$ of an elliptic pseudodifferential operator $\Lambda_{-1} \in \Psi^{-1}(U)$, we have, with some constant $C_{2}>0$,

$$
\left\|X_{0}(u)\right\|_{-\frac{1}{2}}^{2} \leq C_{2}\left(\left|\left(X_{0}(u) \mid A_{0}(u)\right)_{0}\right|+\|u\|_{0}^{2}\right), \quad \forall u \in \mathcal{C}_{0}^{\infty}(U) .
$$

We obtain, with constants $C_{3}, C_{4}>0$,

$$
\begin{gathered}
\left|\left(X_{0}(u) \mid A_{0}(u)\right)\right| \leq\left|\left(P(u) \mid A_{0}(u)\right)_{0}\right|+\left|\sum_{j=1}^{n}\left(L_{j}(u) \mid L_{j}\left(A_{0}(u)\right)\right)_{0}\right| \\
+C_{3}\|u\|\left(\|u\|+\sum_{j=1}^{n}\left\|L_{j}(u)\right\|_{0}\right) \\
\leq\left|\left(P(u) \mid A_{0}(u)\right)_{0}\right|+\sum_{j=1}^{n}\left\|L_{j}(u)\right\|_{0}^{2}+C_{4}\|u\|_{0}^{2}, \\
\forall u \in \mathcal{C}_{0}^{\infty}(U) .
\end{gathered}
$$

To complete the proof, it suffices to show that, if $Y \in \mathfrak{X}(M)$ satisfies, for some $\delta>0$, for some $A_{1}, \ldots, A_{r} \in \Psi^{0}(U)$, and a constant $C^{\prime}>0$, the estimate

$$
\|Y(u)\|_{\delta-1}^{2} \leq C^{\prime}\left(\sum_{h=1}^{r}\left|\left(P(u) \mid A_{h}(u)\right)_{0}\right|+\|u\|_{0}^{2}\right), \quad \forall u \in \mathcal{C}_{0}^{\infty}(U),
$$

and $X \in \mathbb{A}_{\mathfrak{Z}}(M)$, then we have, with some $A_{1}^{\prime}, \ldots, A_{r^{\prime}}^{\prime} \in \Psi^{0}(U)$, and constants $\delta^{\prime}>0, C^{\prime \prime}>0$,

$$
\left\{\begin{array}{r}
\|[X, Y](u)\|_{\delta^{\prime}-1}^{2} \leq C^{\prime \prime}\left(\sum_{h=1}^{r^{\prime}}\left|\left(P(u) \mid A_{h}^{\prime}(u)\right)_{0}\right|+\|u\|_{0}^{2}\right), \\
\forall u \in \mathcal{C}_{0}^{\infty}(U) .
\end{array}\right.
$$

Recall that the estimate (1.13) holds for $Z=X$, and hence

$$
\|X(u)\|_{0}^{2} \leq c_{X}\left(\left|(P(u) \mid u)_{0}\right|+\|u\|_{0}^{2}\right) \quad \forall u \in \mathcal{C}_{0}^{\infty}(U),
$$

with a constant $c_{X}>0$. Then, with positive constants $c_{i}>0$ and a $T \in \Psi^{\delta-1}(U)$, we get

$$
\|[X, Y](u)\|_{\frac{\delta}{2}-1}^{2} \leq c_{0}\left(\left|([X, Y](u) \mid T(u))_{0}\right|+\|u\|_{0}^{2}\right), \quad \forall u \in \mathcal{C}_{0}^{\infty}(U) .
$$


Assuming, as we can, that $\delta \leq \frac{1}{2}$, we obtain

$$
\begin{aligned}
\left|(X Y(u) \mid T(u))_{0}\right| & \leq\left|\left(T^{*}(Y(u)) \mid X(u)\right)_{0}\right|+\left|\left([T, X]^{*}(Y(u)) \mid u\right)_{0}\right|+c_{1}\|u\|_{0}^{2} \\
& \leq c_{2}\|Y(u)\|_{\delta-1}\left(\|X(u)\|_{0}+\|u\|_{0}\right)+c_{1}\|u\|_{0}^{2}, \quad \forall u \in \mathcal{C}^{\infty}(U) .
\end{aligned}
$$

The last term, in view of (4.33), can be estimated by the right hand side of (4.32). Likewise

$$
\begin{gathered}
\left|(Y X(u) \mid T(u))_{0}\right| \leq\left|(X(u) \mid T(Y(u)))_{0}\right|+\left|(X(u) \mid[T, Y](u))_{0}\right|+c_{3}\|u\|_{0}^{2} \\
\leq c_{4}\|X(u)\|_{0}\left(\|Y(u)\|_{\delta-1}+\|u\|_{0}\right)+c_{3}\|u\|_{0}^{2}, \\
\forall u \in \mathcal{C}_{0}^{\infty}(U),
\end{gathered}
$$

and in view of (4.33), also this last term can be estimated by the right hand side of (4.32). The proof is complete.

Remark 4.7. Theorem 4.4 is weaker than the analogous statement in [14] in the case where the $L_{j}$ 's are real. Indeed, for $L_{j} \in \mathfrak{X}(M)$, setting $X_{0}=$ $L_{0}$, our assumption requires that all commutators $\left[L_{j_{1}}, \ldots, L_{j_{r-1}}, L_{j_{r}}\right]$ with $r \in \mathbb{Z}_{+}$, and $0 \leq j_{h} \leq n$, and $j_{h}>0$ for $h<r$, span the tangent space $T_{p} M$. The statement in [14], also proved in [16] and [8], allows $j_{h}=0$ also for $1 \leq h<r$. This motivates us to consider separately the special case where $\mathbb{E}_{\mathfrak{Z}}(M)=\mathfrak{Z}(M)+\overline{\mathfrak{Z}(M)}$ : Theorem 4.8 below generalizes the case where all the $L_{j}$ 's are real.

Theorem 4.8. We keep the notation of Definition 1.11. Assume that

$$
\begin{gathered}
L_{1}, \ldots, L_{n} \in \mathfrak{X}^{\mathbb{C}}(M) \text { generate } \mathfrak{Z}(M), \\
\mathbb{E}_{\mathfrak{Z}}(M) \supset \overline{\mathfrak{Z}(M)}, \\
L_{0} \text { is a real vector field, } \\
L_{n+1} \in \mathfrak{Z}(M)+\overline{\mathfrak{Z}(M)}, \\
a \in \mathcal{C}^{\infty}(M) .
\end{gathered}
$$

Let us define:

$$
\begin{gathered}
\mathbb{A}^{\prime}(M)=\mathbb{A}_{(0)}^{\prime}(M)=\mathbb{A}_{\mathfrak{Z}}(M)+\mathcal{C}^{\infty}(M, \mathbb{R}) L_{0} \\
\mathbb{A}_{(h)}^{\prime}(M)=\left[\mathbb{A}^{\prime}(M), \mathbb{A}_{(h-1)}^{\prime}(M)\right] \text { for } h \geq 1, \\
\mathfrak{T}^{\prime \prime}(M)=\sum_{h=0}^{\infty} \mathbb{A}_{(h)}^{\prime}
\end{gathered}
$$

Then the second order differential operator

$$
P(u)=L_{0}(u)+\sum_{j=1}^{n} \bar{L}_{j} L_{j}(u)+L_{n+1}(u)+a u
$$

is hypoelliptic at all points $p \in M$ where

$$
\left\{X(p) \mid X \in \mathfrak{T}^{\prime \prime}(M)\right\}=T_{p} M .
$$

Proof. We shall prove that, for every $X \in \mathfrak{T}^{\prime \prime}(M)$,

$$
\left\{\begin{array}{c}
\forall U^{\text {open }} \Subset M, \exists \epsilon>0, \exists C>0, \text { s.t. } \\
\|X(u)\|_{\epsilon-1}^{2} \leq C\left(\|\left(P(u)\left\|_{0}^{2}+\right\| u \|_{0}^{2}\right), \quad \forall u \in \mathcal{C}_{0}^{\infty}(U) .\right.
\end{array}\right.
$$


We observe that the proof of Theorem 4.4 shows that, if $Y \in \mathfrak{X}(M)$ satisfies (4.44), and $X \in \mathbb{A}_{\mathfrak{Z}}(M)$, then $[X, Y]$ also satisfies (4.44) (with $\epsilon / 2$ substituting $\epsilon$ ). Thus to show that all $X \in \mathfrak{T}^{\prime \prime}(M)$ satisfy (4.44), it suffices to prove the following

Lemma 4.9. If $Y \in \mathfrak{X}(M)$ satisfies (4.44), then $\left[L_{0}, Y\right]$ also satisfies (4.44).

Proof. We closely follow the argument in [16, p.66-68]. Condition (4.35) means that, for every $U \Subset M$ there is a constant $C_{0}>0$ such that

$$
\sum_{j=1}^{n}\left\|\bar{L}_{j}(u)\right\|_{0}^{2} \leq C_{0}\left(\sum_{j=1}^{n}\left\|L_{j}(u)\right\|_{0}^{2}+\|u\|_{0}^{2}\right), \quad \forall u \in \mathcal{C}_{0}^{\infty}(U) .
$$

Thus, by (4.30), we obtain, with a constant $C_{1}$ that only depends on $U \Subset M$,

$$
\left\{\begin{array}{r}
\sum_{j=1}^{n}\left(\left\|L_{j}(u)\right\|_{0}^{2}+\left\|\bar{L}_{j}(u)\right\|_{0}^{2}\right) \leq C_{1}\left(\left|(P(u) \mid u)_{0}\right|+\|u\|_{0}^{2}\right) \\
\forall u \in \mathcal{C}_{0}^{\infty}(U) .
\end{array}\right.
$$

Let $Y \in \mathfrak{X}(M)$ satisfy (4.44). We have, with $T_{2 \delta-1} \in \Psi^{2 \delta-1}(U)$, and for all $u \in \mathcal{C}_{0}^{\infty}(U)$,

$$
\begin{aligned}
\left\|\left[L_{0}, Y\right](u)\right\|_{\delta-1}^{2} & \leq C_{2}\left(\left|\left(\left[L_{0}, Y\right](u) \mid T_{2 \delta-1}(u)\right)_{0}\right|+\|u\|_{0}^{2}\right) \\
& \leq C_{2}\left(\left|\left(L_{0} Y(u) \mid T_{2 \delta-1}(u)\right)_{0}\right|+\left|\left(Y L_{0}(u) \mid T_{2 \delta-1}(u)\right)_{0}\right|+\|u\|_{0}^{2}\right) .
\end{aligned}
$$

We shall estimate separately each summand inside the parentheses in the last term. In the following $U$ will be a relatively compact open subset of $M$ and all functions $u$ will be smooth and have compact support in some fixed relatively compact open subset of $U$.

We have

$$
P^{*}(u)=-L_{0}(u)+\sum_{j=1}^{n} L_{j}^{*} L_{j}(u)+L_{n+1}^{\prime}(u)+a^{\prime} u,
$$

with $L_{n+1}^{\prime} \in \mathfrak{Z}(M)+\overline{\mathfrak{Z}(M)}$ and $a^{\prime} \in \mathcal{C}^{\infty}(M)$. Hence using (4.46),

$$
\begin{aligned}
& \left|\left(L_{0} Y(u) \mid T_{2 \delta-1}(u)\right)_{0}\right| \leq\left|\left(P^{*} Y(u) \mid T_{2 \delta-1}(u)\right)_{0}\right| \\
& +\sum_{j=1}^{n}\left|\left(L_{j}^{*} L_{j} Y(u) \mid T_{2 \delta-1}(u)\right)_{0}\right|+C_{3}\left(\|u\|_{0}^{2}+\|Y(u)\|_{2 \delta-1}^{2}+\|P(u)\|_{0}^{2}\right) .
\end{aligned}
$$

The term in parenthesis can be estimated by a constant times $\left(\|u\|_{0}^{2}+\right.$ $\|P(u)\|_{0}^{2}$ ), provided we choose a $\delta$ so small that $Y$ satisfies (4.44) in $U$ with some $\epsilon \geq 2 \delta$. For the first summand on the right hand side of (因), we have

$$
\left(P^{*} Y(u) \mid T_{2 \delta-1}(u)\right)_{0}=\left(T_{2 \delta-1}^{*}(Y(u)) \mid P(u)\right)_{0}+\left(Y(u) \mid\left[P, T_{2 \delta-1}\right](u)\right)_{0} .
$$

We obtain

$$
\left|\left(T_{2 \delta-1}^{*}(Y(u)) \mid P(u)\right)_{0}\right| \leq C_{4}\left(\|Y(u)\|_{2 \delta-1}^{2}+\|P(u)\|_{0}^{2}\right) .
$$

The right hand side is bounded by a constant times $\left(\|u\|_{0}^{2}+\|P(u)\|_{0}^{2}\right)$, provided again that (4.44) holds for $Y$ with $\epsilon \geq 2 \delta$. 
For the commutator $\left[P, T_{2 \delta-1}\right]$, we have

$$
\left[P, T_{2 \delta-1}\right]=\sum_{j=1}^{n} T_{2 \delta-1}^{\prime} L_{j}^{*}(u)+T_{2 \delta-1}^{\prime \prime} L_{j}(u)+T_{2 \delta-1}^{\prime \prime \prime},
$$

with $T_{2 \delta-1}^{\prime}, T_{2 \delta-1}^{\prime \prime}, T_{2 \delta-1}^{\prime \prime \prime} \in \Psi^{2 \delta-1}(U)$

Thus, because of (4.46), also the term $\left|\left(Y(u) \mid\left[P, T_{2 \delta-1}\right](u)\right)_{0}\right|$ is bounded by a constant times $\left(\|u\|_{0}^{2}+\|P(u)\|_{0}^{2}\right)$, provided again that (4.44) holds for $Y$ with $\epsilon \geq 2 \delta$.

We have

$$
T_{2 \delta-1}^{*} L_{j}^{*} L_{j}=L_{j}^{*} L_{j} T_{2 \delta-1}^{*}+L_{j} B_{2 \delta-1}^{\prime}+L_{j}^{*} B_{2 \delta-1}^{\prime \prime}+B_{2 \delta-1}^{\prime \prime \prime}
$$

with $B_{2 \delta-1}^{\prime}, B_{2 \delta-1}^{\prime \prime}, B_{2 \delta-1}^{\prime \prime \prime} \in \Psi^{2 \delta-1}(U)$. Thus with a constant $C_{4}>0$,

$$
\begin{aligned}
\left|\left(L_{j}^{*} L_{j} Y(u) \mid T_{2 \delta-1}(u)\right)_{0}\right| & \leq\left|\left(L_{j} T_{2 \delta-1}^{*}(u) \mid L_{j}(u)\right)_{0}\right|+\left|\left(B_{2 \delta-1}^{\prime} Y(u) \mid L_{j}^{*}(u)\right)_{0}\right| \\
& +\left|\left(B_{2 \delta-1}^{\prime \prime} Y(u) \mid L_{j}(u)\right)_{0}\right|+\left|\left(B_{2 \delta-1}^{\prime \prime \prime} Y(u) \mid u\right)_{0}\right| \\
& \leq C_{4}\left(\left\|L_{j} T_{2 \delta-1}^{*} Y(u)\right\|_{0}^{2}+\left\|L_{j}(u)\right\|_{0}^{2}+\left\|\bar{L}_{j}(u)\right\|_{0}^{2}\right. \\
& \left.+\|Y(u)\|_{2 \delta-1}^{2}+\|u\|_{0}^{2}\right) .
\end{aligned}
$$

Therefore, provided again that (4.44) holds for $Y$ with some $\epsilon \geq 2 \delta$, all terms but those of the form $\left\|L_{j} T_{2 \delta-1}^{*}(u)\right\|_{0}^{2}$ are bounded by a constant times $\left(\|u\|_{0}^{2}+\|P(u)\|_{0}^{2}\right)$. Thus we only need to bound the terms $\left\|L_{j} T_{2 \delta-1}^{*}(u)\right\|_{0}^{2}$. We have, by (4.30), with some constant $C_{5}>0$,

$$
\sum_{j=1}^{n}\left\|L_{j} T_{2 \delta-1}^{*} Y(u)\right\|_{0}^{2} \leq C_{5}\left(\left|\left(P\left(T_{2 \delta-1}^{*} Y(u)\right) \mid T_{2 \delta-1}^{*} Y(u)\right)_{0}\right|+\left\|T_{2 \delta-1}^{*} Y(u)\right\|_{0}^{2}\right) .
$$

The last summand inside the parentheses on the right hand side is bounded by a constant times $\left(\|u\|_{0}^{2}+\|P(u)\|_{0}^{2}\right)$, provided again that (4.44) holds for $Y$ with some $\epsilon \geq 2 \delta$. Let us consider the first one. Note that the composition $T_{2 \delta}=T_{2 \delta-1}^{*} \circ Y$ is a pseudodifferential operator in $\Psi^{2 \delta}(U)$. We have the commutation formula

$$
\left[P, T_{2 \delta}\right]=\sum_{j=1}^{n}\left(F_{j_{2 \delta}}^{\prime} L_{j}+F_{j_{2 \delta}}^{\prime \prime} \bar{L}_{j}\right)+F_{2 \delta}^{\prime \prime \prime}
$$

with $F_{j_{2 \delta}}^{\prime}, F_{j_{2 \delta}}^{\prime \prime}, F_{2 \delta}^{\prime \prime \prime} \in \Psi^{2 \delta}(U)$.

Thus

$$
\begin{aligned}
\left(P\left(T_{2 \delta-1}^{*} Y(u)\right) \mid T_{2 \delta-1}^{*} Y(u)\right)_{0}=\left(P\left(T_{2 \delta}(u)\right) \mid T_{2 \delta-1}^{*} Y(u)\right)_{0} \\
=\left(P(u) \mid T_{2 \delta}^{*} T_{2 \delta-1}^{*} Y(u)\right)_{0}+\sum_{j=1}^{n}\left(L_{j}(u) \mid\left[F_{j_{2 \delta}}^{\prime}\right]^{*} T_{2 \delta-1}^{*} Y(u)\right)_{0} \\
\quad+\sum_{j=1}^{n}\left(\bar{L}_{j}(u) \mid\left[F_{j_{2 \delta}}^{\prime \prime}\right]^{*} T_{2 \delta-1}^{*} Y(u)\right)_{0}+\left(u \mid\left[F_{2 \delta}^{\prime \prime \prime}\right]^{*} T_{2 \delta-1}^{*} Y(u)\right)_{0} .
\end{aligned}
$$

Hence we obtain, with some constant $C_{6}>0$,

$$
\begin{array}{r}
\left\|L_{j} T_{2 \delta-1}^{*} Y(u)\right\|_{0}^{2} \leq C_{6}\left(\|P(u)\|_{0}\|Y(u)\|_{4 \delta-1}+\sum_{j=1}^{n}\left(\left\|L_{j}(u)\right\|_{0}^{2}+\left\|\bar{L}_{j}(u)\right\|_{0}^{2}\right)\right. \\
\left.+\|Y(u)\|_{4 \delta-1}^{2}+\|u\|_{0}^{2}\right),
\end{array}
$$


which can be bounded by the right hand side of (4.44), provided $Y$ satisfies (4.44) with $\epsilon \geq 4 \delta$.

Finally we note that

$$
\begin{array}{r}
\left|\left(Y L_{0}(u) \mid T_{2 \delta-1}(u)\right)_{0}\right| \leq C_{7}\left(\left|\left(T_{2 \delta-1}^{*}(u) \mid L_{0} Y(u)\right)_{0}\right|+\left\|L_{0}(u)\right\|_{2 \delta-1}^{2}\right. \\
\left.+\|Y(u)\|_{2 \delta-1}^{2}+\|u\|_{0}^{2}\right) .
\end{array}
$$

Thus, by repeating the discussion above with $T_{2 \delta-1}^{*}$ replacing $T_{2 \delta-1}$, we find that the left hand side of (4.47), provided $Y$ satisfies (4.44) with $\epsilon \geq 4 \delta$, is bounded by a constant times $\left(\|u\|_{0}^{2}+\|P(u)\|_{0}^{2}\right)$. This concludes the proof of the Lemma.

End of the Proof of Theorem 4.8. By the discussion at the beginning of the proof, and by Lemma 4.9, we obtain that for every relatively compact open subset $U$ of $M$, which is contained in the open subset $M^{\prime}$ of $M$, consisting of the points $p$ where (4.43) is satisfied, there are positive constants $\epsilon>0$ and $c_{0}>0$ such that

$$
\left\{\begin{array}{r}
\|u\|_{\epsilon}^{2}+\sum_{j=1}^{n}\left(\left\|L_{j}(u)\right\|_{0}^{2}+\left\|\bar{L}_{j}(u)\right\|_{0}^{2}\right) \leq c_{0}\left(\|u\|_{0}^{2}+\|P(u)\|_{0}^{2}\right), \\
\forall u \in \mathcal{C}_{0}^{\infty}(U) .
\end{array}\right.
$$

One easily shows by recurrence that, if $U$ is a relatively compact open subset of $M$, then there is a positive constant $\epsilon>0$, and, for every real $s \geq 0$ another constant $c_{s} \geq 0$, such that

$$
\left\{\begin{aligned}
\|u\|_{\epsilon+s}^{2}+\sum_{j=1}^{n}\left(\left\|L_{j}(u)\right\|_{s}^{2}+\left\|\bar{L}_{j}(u)\right\|_{s}^{2}\right) \leq c_{0}\left(\|u\|_{0}^{2}+\right. & \left.\|P(u)\|_{s}^{2}\right), \\
& \forall u \in \mathcal{C}_{0}^{\infty}(U) .
\end{aligned}\right.
$$

The hypoellipticity of $P$ in $U$, with a gain of $\epsilon$ derivatives, follows in a standard way from (4.49) (see e.g. [14]).

\section{Applichtions to almost $C R$ manifolds}

In this section we shall consider the case where $M$ is an almost $C R$ manifold of $C R$ dimension $n$ and $C R$ codimension $k$, and $\mathfrak{Z}(M)$ is the distribution of vector fields of type $(0,1)$ on $M$. This means that conditions $(i),(i i)$ and (iii) below are satisfied:

$M$ has real dimension $2 n+k$,

$\mathfrak{Z}(M)$ has constant rank $n$,

$$
\begin{aligned}
& \mathfrak{Z}(M) \cap \overline{\mathfrak{Z}(M)}=\{0\}, \\
& {[\mathfrak{Z}(M), \mathfrak{Z}(M)] \subset \mathfrak{Z}(M) .}
\end{aligned}
$$

When the formal integrability condition (iv) is also satisfied, we say that $M$ is a $C R$ manifold.

When $M$ is an almost $C R$ manifold, it is customary to write $T^{0,1} M$ for the complex bundle with fibers $T_{p}^{0,1} M=Z_{p} M$. The $Z$-morphisms of $₫ 3$ are then the $C R$ maps, i.e. the smooth maps $\phi: N \rightarrow M$ with $d \phi^{\mathbb{C}}\left(T_{q}^{0,1} N\right) \subset T_{\phi(q)}^{0,1} M$ for all $q \in N$. 
Lemma 5.1. If an almost $C R$ manifold $M$ satisfies the higher order Levi form concavity condition at a point $p$, then $M$ is of finite type at $p$.

The next statements clarify in what sense condition (2.32) is a pseudoconcavity condition.

Proposition 5.2. Let $M$ be a CR manifold of hypersurface type, i.e. of $C R$ codimension $k=1$. If $\mathfrak{Z}(M)$ satisfies the higher Levi form concavity condition (2.32) at a point $p$, which is regular for $\mathfrak{Z}(M)$, then $M$ is strictly pseudoconcave at $p$.

Proof. Let $0 \neq \xi \in H_{p}^{0} M$. We want to prove that $\mathcal{L}_{\xi}$ is indefinite. Assume by contradiction that this is not the case. Replacing, if needed, $\xi$ by $(-\xi)$, we can assume that $\mathcal{L}_{\xi} \geq 0$. By assumption (2.32) we can choose $Z_{0} \in \mathfrak{Z}(M)$ and $Z_{1}, \ldots, Z_{r} \in \mathbb{K}_{\mathfrak{Z}}(M) \cup \overline{\mathbb{K}_{\mathfrak{Z}}(M)}$ satisfying

$$
\xi\left(\left[Z_{1}, \ldots, Z_{r}, \bar{Z}_{0}\right]\right) \neq 0 .
$$

We can take $r$ minimal with this property. In particular, we have

$$
\left[Z_{2}, \ldots, Z_{r}, \bar{Z}_{0}\right](p)=Z(p)+\bar{W}(p),
$$

with $Z, W \in \mathfrak{Z}(M)$. Assume that $Z_{1} \in \mathbb{K}_{\mathfrak{Z}}(M)$. Then $\xi\left(\left[Z_{1}, Z\right]\right)=0$, because of the integrability condition $(i v)$, and then, from (函), we have $\mathcal{L}_{\xi}\left(Z_{1}, \bar{W}\right) \neq 0$ and $\mathcal{L}_{\xi}\left(Z_{1}, \bar{Z}_{1}\right)=0$, yielding a contradiction. Likewise, if $Z_{1} \in \overline{\mathbb{K}_{\mathfrak{Z}}(M)}$, we have $\xi\left(\left[Z_{1}, \bar{W}\right]\right)=0$ by (iv), and hence, from (荬), $\mathcal{L}_{\xi}\left(Z, Z_{1}\right) \neq 0$, while $\mathcal{L}_{\xi}\left(\bar{Z}_{1}, Z_{1}\right)=0$, contradicting the assumption that $\mathcal{L}_{\xi} \geq 0$. The proof is complete.

Corollary 5.3. Let $N$ be a generic $C R$ submanifold of a $C R$ manifold $M$ (this means that $T_{p}^{0,1} N=T_{p}^{0,1} M \cap T_{p}^{\mathbb{C}} N$ and the restriction map $H_{p}^{0} M \rightarrow$ $H_{p}^{0} N$ is injective for all $p \in N \subset M$ ). If $M$ is of hypersurface type and $\mathfrak{Z}(N)$ satisfies the higher Levi form concavity condition (2.32) at a point $p_{0}$, regular for $\mathfrak{Z}(M)$, then $M$ is strictly pseudoconcave at $p_{0}$.

Proof. The statement follows from Propositions 3.8 and 5.2 ,

Lemma 5.4. Let $M, N$ be almost $C R$ manifolds, and $\varpi: N \rightarrow M$ a $C R$ map and a smooth submersion. We denote by $\mathfrak{Z}(M)$ and $\mathfrak{Z}(N)$ the distributions of $(0,1)$ vector fields on $M, N$, respectively. If $M$ is strictly pseudoconvex at a point $p_{0}$, i.e. if there is $\xi_{0} \in H_{p_{0}}^{0} M$ with $\mathcal{L}_{\xi_{0}}>0$, and moreover $p_{0}$ is regular for $\mathfrak{Z}(M)$, then the higher Levi form concavity condition (2.32) for $\mathfrak{Z}(N)$ is not satisfied at any point $q_{0} \in \varpi^{-1}\left(p_{0}\right)$.

Proof. Replacing $M$ by an open neighborhood of $p_{0}$ in $M$, we can assume that $M$ is strictly pseudoconvex at all points. Then $\mathbb{K}_{\mathfrak{Z}}(M)=0$, and hence $\mathbb{K}_{\mathfrak{Z}}(N)$ is contained in the complexification of the vertical distribution $\mathfrak{V}^{\varpi}(N)$. Indeed, if $\xi \in H_{p}^{\oplus} M$ and $q \in \varpi^{-1}(p)$, the pullback $\varpi^{*}(q)(\xi)$ belongs to $H_{q}^{\oplus} N$.

Thus if $\eta_{0}=\varpi^{*}\left(q_{0}\right)\left(\xi_{0}\right)$ for $q_{0} \in \varpi^{-1}\left(p_{0}\right)$, then $\eta_{0}$ does not satisfy (2.32). Indeed, $\eta_{0}$ vanishes on the pullback of $\mathfrak{Z}(M)+\overline{\mathfrak{Z}(M)}$, and this distribution is a $\mathfrak{V}^{\varpi}(N)$-module.

Theorem 4.1 and Corollary 4.2 yield 
Theorem 5.5. Let $M$ be an almost $C R$ manifold and assume that condition (1.21) is satisfied by the distribution $\mathfrak{Z}(M)$ of its $(0,1)$ vector fields at all points of $M$. Then

(1) If $E \stackrel{\pi}{\rightarrow} M$ a Hermitian vector bundle on $M$, endowed with a $\mathbb{C}$-linear connection $\nabla$, then all weak solutions $u \in L_{\mathrm{loc}}^{2}(M, E)$ of

$$
\nabla_{Z}(u) \in \mathcal{C}^{\infty}(M, E), \quad \forall Z \in \mathfrak{Z}(M)
$$

are smooth sections of $E \stackrel{\pi}{\rightarrow} M$.

In particular, all $C R$ sections of $E$ (i.e. weak $L_{\text {loc }}^{2}$ solutions of $\nabla_{Z}(u)=0$, for all $\left.Z \in \mathfrak{Z}(M)\right)$ are smooth.

(2) In case $M$ is compact, the space of $C R$ sections of $E$ is a finite dimensional $\mathbb{C}$-linear space.

(3) If $M$ is a compact $C R$ manifold, then the cohomology groups $H_{\bar{\partial}_{M}}^{p, 1}(M)$, for $p=1, \ldots, n+k$, of the tangential Cauchy-Riemann complexes, are Hausdorff.

\section{Subellipticity CONDITIONS For Homogeneous $C R$ MANifolds}

Let $M$ be a $C R$ manifold, homogeneous for the $C R$ action of a Lie group $\mathbf{G}$. Fix a base point $\mathbf{o} \in M$ and denote by

$$
\varpi: \mathbf{G} \ni g \rightarrow g \cdot \mathbf{o} \in M
$$

the associated principal bundle. In [20] we associated to $M$ and the base point $\mathbf{o}$ the $C R$ algebra $(\mathfrak{g}, \mathfrak{q})$, that is the pair consisting of

- the Lie algebra $\mathfrak{g}$ of $\mathbf{G}$,

- the complex Lie subalgebra $\mathfrak{q}$ of $\mathfrak{g}^{\mathbb{C}}=\mathfrak{g}+i \mathfrak{g}$ given by

$$
\mathfrak{q}=\left[d \varpi_{e}\right]_{\mathbb{C}}^{-1}\left(T_{\mathbf{o}}^{0,1} M\right),
$$

where $\left[d \varpi_{e}\right]_{\mathbb{C}}: \mathfrak{g}^{\mathbb{C}} \rightarrow T_{\mathbf{o}}^{\mathbb{C}} M$ is the complexification of the differential $d \varpi_{e}: \mathfrak{g} \rightarrow T_{\mathbf{o}} M$ of $\varpi$ at the identity.

For $X \in \mathfrak{g}$ we denote by $X^{*}$ the left-invariant vector field in $\mathbf{G}$ with $X^{*}(e)=$ $X$. Let

$$
\begin{aligned}
\mathfrak{q}^{*} & =\left\{X^{*}+i Y^{*} \mid X, Y \in \mathfrak{g}, X+i Y \in \mathfrak{q}\right\}, \\
\mathfrak{Z}(\mathbf{G}) & =\mathcal{E}(M) \otimes \mathfrak{q}^{*}=\text { the vector distribution spanned by } \mathfrak{q}^{*} .
\end{aligned}
$$

The following statement is straightforward.

Proposition 6.1. Let $M=\mathbf{G} / \mathbf{G}_{\mathbf{o}}$ be a $\mathbf{G}$-homogeneous $C R$ manifold, $\mathfrak{Z}(M)$ the distribution of $(0,1)$-vector fields on $M$, and $\mathfrak{Z}(\mathbf{G})$ the distribution on $\mathbf{G}$ defined by (6.4). Then

(1) The distributions $\mathfrak{Z}(M)$ and $\mathfrak{Z}(\mathbf{G})$ are regular at all points;

(2) the principal bundle fibration $\mathbf{G} \stackrel{\varpi}{\longrightarrow} M$ is a $Z$-morphism,

$$
\mathfrak{Z}(\mathbf{G})=\left[\varpi^{*} \mathfrak{Z}\right](\mathbf{G})
$$

and conditions (2), (3), (4) of Proposition 3.8 are satisfied.

Proposition 6.1 can be used to reduce the question of the subellipticity of the distribution of $(0,1)$-vector fields of a homogeneous $C R$ manifold to Lie algebra computations. 
Let $(\mathfrak{g}, \mathfrak{q})$ be the $C R$ algebra associated with the $\mathbf{G}$-homogeneous $C R$ manifold $M$ and its base point $\mathbf{o}$. Let

$$
\mathfrak{t}^{0}=H_{e}^{0} \mathbf{G}=\left\{\xi \in \mathfrak{g}^{*} \mid \xi(\operatorname{Re}(Z))=0, \forall Z \in \mathfrak{q}\right\} .
$$

To each $\xi \in \mathfrak{t}^{0}$ we associate the Levi form

$$
\mathcal{L}_{\xi}(Z, \bar{W})=i \xi([Z, \bar{W}]), \text { for } Z, W \in \mathfrak{q} .
$$

We also set

$$
\begin{aligned}
\mathfrak{t}^{\oplus} & =\left\{\xi \in \mathfrak{t}^{0} \mid \mathcal{L}_{\xi} \geq 0\right\}, \\
\mathfrak{k}_{\mathfrak{q}} & =\left\{Z \in \mathfrak{q} \mid \mathcal{L}_{\xi}(Z, \bar{Z})=0, \quad \forall \xi \in \mathfrak{t}^{\oplus}\right\} .
\end{aligned}
$$

Since all points of $M$ and of $\mathbf{G}$ are regular for $\mathfrak{Z}(M)$ and $\mathfrak{Z}(\mathbf{G})$, respectively, we obtain from Proposition 2.13:

Lemma 6.2. Let $(\mathfrak{g}, \mathfrak{q})$ be a $C R$ algebra. The set $\mathfrak{k}_{\mathfrak{q}}$ is a linear subspace of $\mathfrak{q}$ and is equal to the set

$$
\left\{Z_{0} \in \mathfrak{q} \mid \exists Z_{1}, \ldots, Z_{r} \in \mathfrak{q}, \text { s.t. } \sum_{j=0}^{r}\left[Z_{j}, \bar{Z}_{j}\right] \in \mathfrak{q}+\overline{\mathfrak{q}}\right\} .
$$

The elements

$$
Z^{*}=(\operatorname{Re} Z)^{*}+i(\operatorname{Im} Z)^{*}, \quad \text { for } Z \in \mathfrak{k}_{\mathfrak{q}},
$$

generate the distribution $\mathbb{K}_{\mathfrak{Z}}(\mathbf{G})$, that is equal to $\Theta_{\mathfrak{Z}}(\mathbf{G})$.

As a consequence of Propositions 2.16, 6.1 and Lemma 6.2 we have

Proposition 6.3. Let $M=\mathbf{G} / \mathbf{G}_{\mathbf{o}}$ be a homogeneous $C R$ manifold and let $(\mathfrak{g}, \mathfrak{q})$ be the $C R$ algebra associated with $M$ and the base point $\mathbf{o}$. We denote by $\mathfrak{Z}(M)$ the distribution of $(0,1)$-vector fields on $M$. Then the following are equivalent.

(1) $\mathfrak{Z}(M)$ satisfies condition (2.32).

(2) $\mathfrak{Z}(M)$ satisfies condition (1.21).

(3) $\mathfrak{Z}(\mathbf{G})$ satisfies condition (2.32).

(4) $\mathfrak{Z}(\mathbf{G})$ satisfies condition (1.21).

$(5)(\mathfrak{g}, \mathfrak{q})$ satisfies the condition:

$$
\left\{\begin{array}{c}
\forall \xi \in \mathfrak{t}^{\oplus} \backslash\{0\}, \quad \exists Z_{0} \in \mathfrak{q} \text { and } Z_{1}, \ldots, Z_{r} \in \mathfrak{k}_{\mathfrak{q}} \cup \overline{\mathfrak{k}}_{\mathfrak{q}} \\
\text { s.t. } \quad i \xi\left(\left[Z_{1}, \ldots, Z_{r}, \bar{Z}_{0}\right]\right) \neq 0 .
\end{array}\right.
$$

\section{ORBits OF A REAL FORM IN A COMPLEX FLAG MANIFOLD}

In this section we investigate the subellipticity of the distribution of the $(0,1)$-vector fields of the homogeneous $C R$ manifolds which are real orbits of real forms in complex flag manifolds. The study of their $C R$ geometry has been already started in [1, 2], to which we refer for the complete explanation of many details. 
7.1. Complex flag manifolds and orbits of a real form. We recall that a complex flag manifold is a closed complex projective variety, that is a coset space of a connected semisimple complex Lie group $\mathbf{G}^{\mathbb{C}}$ with respect to a complex parabolic subgroup.

A real form $\mathbf{G}$ of $\mathbf{G}^{\mathbb{C}}$ is a real Lie subgroup of $\mathbf{G}^{\mathbb{C}}$ whose Lie algebra $\mathfrak{g}$ is a real form of $\mathfrak{g}^{\mathbb{C}}$, i.e. such that $\mathfrak{g} \subset \mathfrak{g}^{\mathbb{C}}$ and $\mathfrak{g}^{\mathbb{C}}=\mathfrak{g}+i \mathfrak{g}$. We shall write $\bar{Z}$ for the conjugate of an element $Z \in \mathfrak{g}^{\mathbb{C}}$ with respect to the real form $\mathfrak{g}$. The left action of $\mathbf{G}$ decomposes $F$ into a finite set of $\mathbf{G}$-orbits (see 24]). All $\mathbf{G}$-orbits $M=\mathbf{G} \cdot \mathbf{o}$ are generically embedded $C R$ submanifolds of $F$. It turns out that $F$ and also $M$, if we take $\mathbf{G}$ connected, are completely determined by the Lie algebra $\mathfrak{g}^{\mathbb{C}}$, by its real form $\mathfrak{g}$, and by the complex parabolic Lie subalgebra $\mathfrak{q} \subset \mathfrak{g}^{\mathbb{C}}$ of the isotropy subgroup $\mathbf{Q}$ at o. Thus we shall write $M=M(\mathfrak{g}, \mathfrak{q})$, for the homogeneous $C R$ manifold $M$. We denote by $\mathbf{G}_{\mathbf{o}}=\mathbf{Q} \cap \mathbf{G}$ the isotropy subgroup at the base point $\mathbf{o}$ and by $\mathbf{G} \underset{\mathbf{G}_{\mathbf{o}}}{\stackrel{\varpi}{\longrightarrow}} M$ the principal $\mathbf{G}_{\mathbf{o}}$-bundle $\varpi: \mathbf{G} \ni g \rightarrow g \cdot \mathbf{o} \in M$.

The Lie algebra $\mathfrak{g}_{\mathbf{o}}$ of $\mathbf{G}_{\mathbf{o}}$ contains a Cartan subalgebra $\mathfrak{h}$ of $\mathfrak{g}$ (see e.g. $[24,1,2])$. Its complexification $\mathfrak{h}^{\mathbb{C}}=\mathfrak{h}+i \mathfrak{h}$ is a Cartan subalgebra of $\mathfrak{g}^{\mathbb{C}}$. Let $\mathcal{R}$ be the set of roots of $\mathfrak{g}^{\mathbb{C}}$ with respect to $\mathfrak{h}^{\mathbb{C}}$, and $\mathfrak{g}_{\alpha}^{\mathbb{C}}=\left\{Z \in \mathfrak{g}^{\mathbb{C}} \mid[H, Z]=\right.$ $\left.\alpha(H) Z, \forall H \in \mathfrak{h}^{\mathbb{C}}\right\}$ the eigenspace corresponding to the root $\alpha \in \mathcal{R}$. Since $\mathfrak{h}^{\mathbb{C}} \subset \mathfrak{q}$, the subalgebra $\mathfrak{q}$ is $\operatorname{ad}_{\mathfrak{g}^{\mathbb{C}}}\left(\mathfrak{h}^{\mathbb{C}}\right)$-invariant. Hence we have

$$
\begin{aligned}
\mathfrak{q} & =\mathfrak{h}^{\mathbb{C}} \oplus \sum_{\alpha \in \mathcal{Q}} \mathfrak{g}_{\alpha}^{\mathbb{C}}, \quad \text { for } \\
\mathcal{Q} & =\left\{\alpha \in \mathcal{R} \mid \mathfrak{g}_{\alpha}^{\mathbb{C}} \subset \mathfrak{q}\right\} .
\end{aligned}
$$

To say that $\mathfrak{q}$ is parabolic means that $\mathcal{Q}$ contains a positive system of roots $\mathcal{R}^{+}$. Let $\prec$ be a corresponding partial order on the linear span $\mathfrak{h}_{\mathbb{R}}^{*}$ of $\mathcal{R}$, and $\mathcal{B}=\left\{\alpha_{1}, \ldots, \alpha_{\ell}\right\}$ the set of simple positive roots in $\mathcal{R}^{+}$. Every root $\alpha \in \mathcal{R}$ can be written in a unique way as a linear combination with integral coefficients of the elements of $\mathcal{B}$ :

$$
\alpha=\sum_{j=1}^{\ell} k_{j} \alpha_{j},
$$

where all $k_{j}$ 's are either $\geq 0$, or $\leq 0$, according to whether $\alpha$ is positive or negative with respect to $\prec$. We define the support of $\alpha$ to be the set

$$
\operatorname{supp}(\alpha)=\left\{\alpha_{j} \in \mathcal{B} \mid k_{j} \neq 0\right\}
$$

Let

$$
\Phi=\{\alpha \in \mathcal{B} \mid-\alpha \notin \mathcal{Q}\}
$$

be the set of simple roots $\alpha$ whose opposite $(-\alpha)$ does not belong to $\mathcal{Q}$. Then

$$
\mathcal{Q}=\mathcal{R}^{+} \cup\{\alpha \prec 0 \mid \operatorname{supp}(\alpha) \cap \Phi=\emptyset\} .
$$

Since $\mathcal{Q}$ is completely determined by $\Phi$, we shall write $\mathcal{Q}_{\Phi}, \mathfrak{q}_{\Phi}, \mathbf{Q}_{\Phi}$ for the parabolic set of roots, the complex parabolic subalgebra and the complex parabolic subgroup, respectively, that are attached to any special choice of 
the subset $\Phi$ of $\mathcal{B}$. We shall also introduce the notation

$$
\begin{aligned}
& \mathcal{Q}_{\Phi}^{n}=\{\alpha \succ 0 \mid \operatorname{supp}(\alpha) \cap \Phi \neq \emptyset\} \\
& \mathcal{Q}_{\Phi}^{r}=\{\alpha \mid \alpha,-\alpha \in \mathcal{Q}\}=\{\alpha \in \mathcal{R} \mid \operatorname{supp}(\alpha) \cap \Phi=\emptyset\} .
\end{aligned}
$$

The conjugation in $\mathfrak{g}^{\mathbb{C}}$ induced by the real form $\mathfrak{g}$ defines, by duality, a conjugation $\alpha \rightarrow \bar{\alpha}$ in $\mathcal{R}$. We partition $\mathcal{R}$ into three subsets:

$$
\begin{aligned}
\mathcal{R}_{\mathrm{re}} & =\{\alpha \in \mathcal{R} \mid \bar{\alpha}=\alpha\} & & \text { real roots, } \\
\mathcal{R}_{\mathrm{im}} & =\{\alpha \in \mathcal{R} \mid \bar{\alpha}=-\alpha\} & & \text { imaginary roots, } \\
\mathcal{R}_{\mathrm{cx}} & =\{\alpha \in \mathcal{R} \mid \bar{\alpha} \neq \pm \alpha\} & & \text { complex roots. }
\end{aligned}
$$

The Cartan subalgebra $\mathfrak{h}$ is invariant under a Cartan involution $\vartheta$ of $\mathfrak{g}$, whose set of fixed points $\mathfrak{k}$ is a maximal compact Lie subalgebra of $\mathfrak{g}$. With $\mathfrak{p}=\{X \mid \vartheta(X)=-X\}$, we have that for imaginary $\alpha$, the eigenspace $\mathfrak{g}_{\alpha}^{\mathbb{C}}$ is contained either in $\mathfrak{k}^{\mathbb{C}}=\mathfrak{k}+i \mathfrak{k}$, or in $\mathfrak{p}^{\mathbb{C}}=\mathfrak{p}+i \mathfrak{p}$. In the first case we say that $\alpha$ is a compact root. Compact roots form a root subsystem $\mathcal{R}_{\bullet}$ of $\mathcal{R}$.

The G-homogeneous $C R$ structure of $M$ is defined, as in 96 , by assigning the subspace

$$
T_{\mathbf{o}}^{0,1}=d \varpi(e)(\mathfrak{q}) .
$$

Here $e$ is the identity of $\mathbf{G}$ and we denote by the same symbol $d \varpi(e)$ the complexification of the differential $d \varpi(e): \mathfrak{g}=T_{e} \mathbf{G} \rightarrow T_{\mathbf{o}} M$.

For each $Z \in \mathfrak{g}^{\mathbb{C}}$ we can consider the fundamental vector field $Z^{*} \in$ $\mathfrak{X}^{\mathbb{C}}(M)$. Its real and imaginary parts are the infinitesimal generators of the flows associated to the left translations by $\exp (t \operatorname{Re}(Z))$ and $\exp (t \operatorname{Im}(Z))$, respectively.

Fix a Chevalley basis $\left\{1 H_{\alpha} \mid \alpha \in \mathcal{B}\right\} \cup\left\{Z_{\alpha} \mid \alpha \in \mathcal{R}\right\}$ of $\mathfrak{g}^{\mathbb{C}}$. The restrictions to $\mathbf{G}$ of the vector fields $H_{\alpha}$, for $\alpha \in \mathcal{B}$, and $Z_{\alpha}$, for $\alpha \in \mathcal{Q}_{\Phi}$, form a basis for the pullback $\mathfrak{Z}(\mathbf{G})$ of $\mathfrak{Z}(M)$.

Since $\mathfrak{g}^{\mathbb{C}}$ is semisimple, the Killing form $\kappa_{\mathfrak{g}^{\mathbb{C}}}$ is nondegenerate. Thus, as in [1, $\S 13$, we can use the Killing form to identify the complexification $\mathfrak{t}^{0 \mathbb{C}}$ of the space $\mathfrak{t}^{0}$ of (6.6) with the linear span of

$$
Z_{\alpha} \quad \text { for } \quad \alpha \in \mathcal{Q}_{\Phi}^{n} \cap \overline{\mathcal{Q}}_{\Phi}^{n} .
$$

This is obtained by associating to $Z_{\alpha}$ the linear form

$$
f_{\alpha}: \mathfrak{g}^{\mathbb{C}} \ni Z \rightarrow \kappa_{\mathfrak{g}^{\mathbb{C}}}\left(Z_{-\alpha}, Z\right)=\operatorname{trace}\left(\operatorname{ad}_{\mathfrak{g}^{\mathbb{C}}}\left(Z_{-\alpha}\right) \circ \operatorname{ad}_{\mathfrak{g}^{\mathbb{C}}}(Z)\right) \in \mathbb{C} .
$$

Correspondingly we obtain the complexified Levi forms

$$
\mathbf{L}_{\alpha}(Z, \bar{W})=i f_{\alpha}([Z, \bar{W}]), \text { for } Z, W \in \mathfrak{q}_{\Phi} .
$$

When $\alpha \in \mathcal{R}_{\text {re }} \cap \mathcal{Q}$, it actually corresponds to a Levi form (2.7) at $\mathbf{o}$. The intersection $\mathcal{Q}_{\Phi}^{n} \cap \overline{\mathcal{Q}}_{\Phi}^{n}$ does not contain imaginary roots. To a pair of

\footnotetext{
1 This means that $Z_{\alpha} \in \mathfrak{g}_{\alpha}^{\mathbb{C}}$ for all $\alpha \in \mathcal{R}$ and that, for $\alpha \in \mathcal{B}$, we also have $\left[H_{\alpha}, Z_{\alpha}\right]=$ $2 Z_{\alpha},\left[H_{\alpha}, Z_{-\alpha}\right]=-2 Z_{-\alpha},\left[Z_{\alpha}, Z_{-\alpha}\right]=-H_{\alpha}$; and moreover that the linear map defined by $H_{\alpha} \rightarrow-H_{\alpha}$ and $Z_{\alpha} \rightarrow Z_{-\alpha}$ is an involutive automorphism of the Lie algebra $\mathfrak{g}^{\mathbb{C}}$. Moreover, $Z_{\alpha} \in \mathfrak{g}$ when $\alpha \in \mathcal{R}_{\text {re }}$ and, if $\alpha \in \mathcal{R}_{\text {im }}$, we have $\bar{Z}_{\alpha}=Z_{-\alpha}$ when $\alpha$ is compact and $\bar{Z}_{\alpha}=-Z_{-\alpha}$ when $\alpha$ is not compact. In general, $Z_{\bar{\alpha}}=t_{\alpha} \bar{Z}_{\alpha}$, with $t_{\alpha}= \pm 1$, for all roots $\alpha \in \mathcal{R}$, and we can take $t_{\alpha}=1$ when $\alpha \in \mathcal{R}_{\text {re }}$ (see e.g. [5]).
} 
complex roots $\alpha, \bar{\alpha} \in \mathcal{Q}_{\Phi}^{n} \cap \overline{\mathcal{Q}}_{\Phi}^{n}$, correspond the two Hermitian symmetric forms obtained by polarization from the Hermitian quadratic forms

$$
\begin{aligned}
{\left[\operatorname{Re} \mathbf{L}_{\alpha}\right](Z, \bar{Z}) } & =\frac{i}{2}\left(f_{\alpha}([Z, \bar{Z}])-\overline{f_{\alpha}([Z, \bar{Z}])}\right), \\
{\left[\operatorname{Im} \mathbf{L}_{\alpha}\right](Z, \bar{Z}) } & =\frac{1}{2}\left(f_{\alpha}([Z, \bar{Z}])+\overline{f_{\alpha}([Z, \bar{Z}])}\right) .
\end{aligned}
$$

Note that $L_{\alpha}(Z, \bar{W})= \pm \overline{L_{\bar{\alpha}}(W, \bar{Z})}$, when $Z_{\bar{\alpha}}$ equals $\pm \bar{Z}_{\alpha}$, respectively. Thus each Levi form can be written as a linear combination of the $\mathbf{L}_{\beta}$ 's for $\beta \in$ $\mathcal{Q}_{\Phi}^{n} \cap \overline{\mathcal{Q}}_{\Phi}^{n}$, the coefficients of $\mathbf{L}_{\beta}$ and $\mathbf{L}_{\bar{\beta}}$, for $\beta \in \mathcal{Q}_{\Phi}^{n} \cap \overline{\mathcal{Q}}_{\Phi}^{n} \cap \mathcal{R}_{\mathrm{cx}}$ being either conjugate or anticonjugate according to the sign in the equality $Z_{\bar{\beta}}= \pm \bar{Z}_{\beta}$.

\subsection{Semidefinite Levi forms.}

Lemma 7.1. Let $\mathcal{R}$ be a root system. There exist no triples $\alpha, \beta, \gamma \in \mathcal{R}$ with

$$
\left\{\begin{array}{c}
\alpha+\bar{\alpha}, \beta+\bar{\beta}, \gamma+\bar{\gamma} \in \mathcal{R}, \\
\alpha+\bar{\alpha} \neq \beta+\bar{\beta}, \quad \alpha+\bar{\beta}=\gamma+\bar{\gamma}
\end{array}\right.
$$

Proof. Note that $\alpha, \beta, \gamma$ belong to the same irreducible component of $\mathcal{R}$. Thus we may as well assume that $\mathcal{R}$ is irreducible. We argue by contradiction.

The root subsystem $\mathcal{R}^{\prime}$ generated by $\{\alpha+\bar{\alpha}, \beta+\bar{\beta}, \gamma+\bar{\gamma}\}$ is of type $\mathrm{B}_{2}$. Indeed, it is not of type $\mathrm{G}_{2}$ because it is a proper subsystem of a larger root system, since all roots in $\mathcal{R}^{\prime}$ are real, while the fact that $\alpha+\bar{\alpha}$ is a root implies that $\mathcal{R}_{\mathrm{cx}}$ is not empty. Moreover, from $(\alpha+\bar{\alpha})+(\beta+\bar{\beta})=2(\gamma+\bar{\gamma})$, we deduce that $\mathcal{R}^{\prime}$ has rank 2 and contains roots of different lengths. Thus we can choose a basis $\left\{\mathrm{e}_{j}\right\}$ in $\mathfrak{h}_{\mathbb{R}}^{*}$ such that

$$
\alpha+\bar{\alpha}=2 \mathrm{e}_{1}, \quad \beta+\bar{\beta}=2 \mathrm{e}_{2}, \quad \gamma+\bar{\gamma}=\mathrm{e}_{1}+\mathrm{e}_{2},
$$

and, furthermore, that

$$
\alpha=\mathrm{e}_{1}+a \mathrm{e}_{3}, \quad \beta=\mathrm{e}_{2}+a \mathrm{e}_{3}, \quad \gamma=(1 / 2) \mathrm{e}_{1}+(1 / 2) \mathrm{e}_{2}+b \mathrm{e}_{3}+c \mathrm{e}_{4}
$$

for some $a, b, c \in \mathbb{R}$, and $a>0$.

Let $\langle\alpha \mid \beta\rangle=2(\alpha \mid \beta) /(\alpha \mid \alpha)$. Recall that, if $\alpha_{1}, \alpha_{2} \in \mathcal{R}$ are not proportional, then $\left(\left\langle\alpha_{1} \mid \alpha_{2}\right\rangle\left\langle\alpha_{2} \mid \alpha_{1}\right\rangle\right) \in\{0,1,2,3\}$. Since

$$
\langle\alpha \mid \gamma+\bar{\gamma}\rangle\langle\gamma+\bar{\gamma} \mid \alpha\rangle=\frac{2}{1+a^{2}}
$$

we have $a=1$, that is $\alpha=\mathrm{e}_{1}+\mathrm{e}_{3}$ and $\beta=\mathrm{e}_{2}+\mathrm{e}_{3}$. Then

$$
\langle\gamma \mid \alpha+\bar{\alpha}\rangle\langle\alpha+\bar{\alpha} \mid \gamma\rangle=\frac{1}{(1 / 2)+b^{2}+c^{2}}
$$

implies that $b^{2}+c^{2}=1 / 2$. Hence $\|\gamma\|^{2}=1$. This gives a contradiction, because $\|\alpha\|^{2}=2$ and $\|\alpha+\bar{\alpha}\|^{2}=4$, and at most two different root lengths are allowed in an irreducible root system.

As a consequence of Proposition 6.3 we obtain

Proposition 7.2. Let $M\left(\mathfrak{g}, \mathfrak{q}_{\Phi}\right)$ be a $\mathbf{G}$-orbit in the complex flag manifold $F$, and $\mathfrak{Z}(M)$ the distribution of its $(0,1)$-vector fields. Set

$$
\mathcal{K}_{\Phi}=\left\{\alpha \in \mathcal{R} \mid \mathfrak{g}_{\alpha}^{\mathbb{C}} \subset \mathfrak{k}_{\mathfrak{q}_{\Phi}}\right\} .
$$


Then

$$
\mathcal{K}_{\Phi}=\left\{\begin{array}{l|l}
\alpha \in \mathcal{Q} & \begin{array}{l}
\text { either }-(\alpha+\bar{\alpha}) \notin \mathcal{Q} \\
\text { or } \mathbf{L}_{\alpha+\bar{\alpha}} \text { is indefinite }
\end{array}
\end{array}\right\}
$$

and we have

$$
\mathfrak{k}_{\mathfrak{q}_{\Phi}}=\mathfrak{h}^{\mathbb{C}} \oplus \sum_{\alpha \in \mathcal{K}_{\Phi}} \mathfrak{g}_{\alpha}^{\mathbb{C}}
$$

A sufficient condition, in order that $\mathfrak{Z}(M)$ satisfy the higher Levi form concavity condition (2.32), is that

$$
\left\{\begin{array}{l}
\text { for each } \gamma \in \mathcal{Q}_{\Phi}^{n} \text { with } \gamma=\bar{\gamma} \text { and } \mathbf{L}_{\gamma} \geq 0, \\
\text { there exist } \alpha_{0} \in \overline{\mathcal{Q}}_{\Phi}, \text { and } \alpha_{1}, \ldots, \alpha_{r} \in \mathcal{K}_{\Phi} \cup \overline{\mathcal{K}}_{\Phi} \text { s.t. } \\
\sum_{j=0}^{h} \alpha_{j} \in \mathcal{R} \text { for } 1 \leq h \leq r,-\gamma=\sum_{j=0}^{r} \alpha_{j} .
\end{array}\right.
$$

Proof. Denote by $\mathcal{H}^{\oplus}$ the set of real roots $\gamma \in \mathcal{Q}_{\Phi}^{n} \cap \overline{\mathcal{Q}}_{\Phi}^{n}$ such that the corresponding Levi form $\mathbf{L}_{\gamma}$ is semidefinite.

Consider any Levi form $\mathbf{L}=\sum_{\beta \in \mathcal{Q}_{\Phi}^{n} \cap \overline{\mathcal{Q}}_{\Phi}^{n}} c_{\beta} \mathbf{L}_{\beta}$, with $c_{\bar{\beta}}= \pm \bar{c}_{\beta}$. We claim that if $\mathbf{L}$ is semidefinite then

$$
\bigcap_{\gamma \in \mathcal{H}^{\oplus}} \operatorname{ker} \mathbf{L}_{\gamma} \subset \operatorname{ker} \mathbf{L} .
$$

This claim implies (7.16) and (7.17), from which the last statement follows.

With respect to the basis $\left\{Z_{\alpha}\right\}_{\alpha \in \mathcal{Q} \backslash \overline{\mathcal{Q}}}$, the complexified Levi forms $\mathbf{L}_{\beta}$ have mutually non overlapping nonzero entries, and each of them has at most one nonzero entry in each row (or column). Note that, for $\beta \in \mathcal{Q}_{\Phi}^{n} \cap \overline{\mathcal{Q}}_{\Phi}^{n} \cap \mathcal{R}_{\mathrm{cx}}$, the matrix of $\mathbf{L}_{\beta}$ has no diagonal entries. Partition $\mathcal{Q}_{\Phi}^{n} \cap \overline{\mathcal{Q}}_{\Phi}^{n}$ into the following subsets:

$$
\begin{aligned}
& \mathcal{H}_{0}=\left\{\beta \in \mathcal{Q}_{\Phi}^{n} \cap \overline{\mathcal{Q}}_{\Phi}^{n} \cap \mathcal{R}_{\text {re }} \mid \mathbf{L}_{\beta} \text { is semidefinite (hence diagonal) }\right\}, \\
& \mathcal{H}_{1}=\left\{\beta \in \mathcal{Q}_{\Phi}^{n} \cap \overline{\mathcal{Q}}_{\Phi}^{n} \cap \mathcal{R}_{\text {re }} \mid \mathbf{L}_{\beta} \text { is indefinite and diagonal }\right\}, \\
& \mathcal{H}_{2}=\left\{\beta \in \mathcal{Q}_{\Phi}^{n} \cap \overline{\mathcal{Q}}_{\Phi}^{n} \mid \mathbf{L}_{\beta} \text { has no nonzero entry on the diagonal }\right\}, \\
& \mathcal{H}_{3}=\left\{\beta \in \mathcal{Q}_{\Phi}^{n} \cap \overline{\mathcal{Q}}_{\Phi}^{n} \cap \mathcal{R}_{\mathrm{re}} \mid \mathbf{L}_{\beta} \quad\right. \text { is indefinite and has nonzero } \\
& \text { entries on and off the diagonal }\}
\end{aligned}
$$

If a coefficient $c_{\beta}$ in the decomposition of $\mathbf{L}$ is different from zero for some $\beta \in \mathcal{H}_{1}$, then $\mathbf{L}$ is indefinite. The same is true for $\beta \in \mathcal{H}_{3}$. Indeed, $\beta$ is real and there exists $\gamma$ such that $\beta=\gamma+\bar{\gamma}$ because $\mathbf{L}_{\beta}$ has a nonzero diagonal entry. Moreover, there are roots $\alpha, \alpha^{\prime} \in \mathcal{Q}$ such that $\beta=\alpha+\bar{\alpha}^{\prime}$ because $\mathbf{L}_{\beta}$ has a nonzero entry off of the main diagonal. By Lemma 7.1, the restriction of $\mathbf{L}_{\beta}$ to $\mathfrak{g}^{\alpha}+\mathfrak{g}^{\alpha^{\prime}}$ is indefinite. Hence the only possible nonzero coefficients are those corresponding to roots in $\mathcal{H}_{0}=\mathcal{H}^{\oplus}$ and $\mathcal{H}_{2}$. The statement then follows from the following statement about Hermitian matrices:

Let $A=\left(a_{i, j}\right)_{1 \leq i, j \leq \ell}$ be a Hermitian symmetric matrix with $a_{i, i}=0$ for $i=1, \ldots, \ell$, and $D=\operatorname{diag}\left(d_{1,1}, \ldots, d_{\ell, \ell}\right)$. If $D+A$ is semidefinite then $\operatorname{ker} D \subset \operatorname{ker} D+A$.

By using Proposition 3.8 we obtain

Proposition 7.3. Let $\mathfrak{g}$ be a semisimple real Lie algebra, $\mathfrak{h}$ a Cartan subalgebra of $\mathfrak{g}$ and $\mathcal{B}$ a system of simple roots of $\mathcal{R}^{+}$. Let $\Psi \subset \Phi \subset \mathcal{B}$. 
If $\mathfrak{Z}\left(M\left(\mathfrak{g}, \mathfrak{q}_{\Phi}\right)\right)$ is subelliptic, then $\mathfrak{Z}\left(M\left(\mathfrak{g}, \mathfrak{q}_{\Psi}\right)\right)$ is also subelliptic.

Proof. The inclusions $\mathfrak{q}_{\Phi} \subset \mathfrak{q}_{\Psi}$ and $\mathbf{Q}_{\Phi} \subset \mathbf{Q}_{\Psi}$ induce a smooth submersion $M\left(\mathfrak{q}, \mathfrak{q}_{\Phi}\right) \rightarrow M\left(\mathfrak{q}, \mathfrak{q}_{\Psi}\right)$, that is also a $C R$ map and satisfies the hypotheses of Proposition 3.8 .

7.3. Minimal orbits. In [24] it is shown that there is a unique $\mathbf{G}$ orbit in $F$ (the minimal orbit) that is compact. It is connected and has minimal dimension. Minimal orbits have been studied, from the point of view of $C R$ geometry, in [1]. If $(\mathfrak{g}, \mathfrak{q})$ is the $C R$ algebra of a minimal orbit $M$, then $\mathfrak{g}_{\mathbf{o}}$ contains a maximally vectorial Cartan subalgebra $\mathfrak{h}$ of $\mathfrak{g}$. This choice of $\mathfrak{h}$ is equivalent to the fact that $\mathcal{R}_{\mathrm{im}}=\mathcal{R}_{\bullet}$, i.e. that all imaginary roots are compact (see [3]).

The fact that $M=M(\mathfrak{g}, \mathfrak{q})$ is minimal is then equivalent to the possibility of choosing the positive root system $\mathcal{R}^{+} \subset \mathcal{Q}$ in such a way that

$$
\text { for } \alpha \in \mathcal{R}_{\mathrm{cx}} \quad \alpha \succ 0 \Longleftrightarrow \bar{\alpha} \succ 0 \text {. }
$$

This leads to a complete classification of the minimal orbits in terms of cross-marked Satake diagrams (see e.g. [1]), i.e. by systems $\Phi \subset \mathcal{B}$, where $\mathcal{B}$ are the simple roots of a positive system $\mathcal{R}^{+}$satisfying (17.19). Using these $\Phi$ 's, we give below a complete classification of the minimal orbits for which the distribution of $(0,1)$ vector fields satisfies the higher order Levi concavity condition (2.32).

When $\mathfrak{g}$ decomposes into a sum $\mathfrak{g}=\mathfrak{g}^{(1)} \oplus \cdots \oplus \mathfrak{g}^{(\ell)}$ of simple ideals, the $C R$ manifold $M(\mathfrak{g}, \mathfrak{q})$ is a Cartesian product $M\left(\mathfrak{g}^{(1)}, \mathfrak{q}^{(1)}\right) \times \cdots \times M\left(\mathfrak{g}^{(\ell)}, \mathfrak{q}^{(\ell)}\right)$, (see [1, p.490]). Thus we can reduce the question of the validity of the higher order Levi form concavity condition, and also of the subellipticity and hypoellipticity of $\mathfrak{Z}(M(\mathfrak{g}, \mathfrak{q}))$, to the case where $\mathfrak{g}$ is a simple real Lie algebra. Namely, this property will be valid for $M(\mathfrak{g}, \mathfrak{q})$ if, and only if, it is valid for each factor $M\left(\mathfrak{g}^{(h)}, \mathfrak{q}^{(h)}\right)$, for $1 \leq h \leq \ell$.

Thus we state the following classification theorem for the case of a simple $\mathfrak{g}$.

For the Satake diagrams characterizing the different simple real Lie algebras, their labels and those of the roots of a simple positive system, we refer to [10], or to the Appendix in [1].

Theorem 7.4. Let $\left(\mathfrak{g}, \mathfrak{q}_{\Phi}\right)$ be the $C R$ algebra of a minimal orbit $M$, with $\mathfrak{g}$ simple and assume that $M$ is a $C R$ manifold of finite type in the sense of 4 . Then $\mathfrak{Z}(M)$ satisfies the higher order Levi form concavity condition (2.32) if and only if one of the following conditions is satisfied

(1) $\mathfrak{g}$ is either of the complex type, or compact, or of one of the real types AII, AIIIb, B, CIIb, DI, DII, DIIIa, EII, EIV, EVI, EVII, EIX;

(2) $\mathfrak{g} \simeq \mathfrak{s u}(p, q)$ is of type AIIIa - AIV and $\Phi \cap\left\{\alpha_{p}, \alpha_{q}\right\}=\emptyset$;

(3) $\mathfrak{g} \simeq \mathfrak{s p}(p, \ell-p)$, with $2 p<\ell$, is of type CIIa and either $\Phi$ is all contained in $\left\{\alpha_{2 j-1} \mid 1 \leq j<p\right\} \cup\left\{\alpha_{j} \mid 2 p<j \leq \ell\right\}$, or $\Phi$ is all contained in $\left\{\alpha_{2 j-1} \mid 1 \leq j \leq p\right\}$

(4) $\mathfrak{g} \simeq \mathfrak{s o}^{*}(2 \ell)$, with $\ell \in 2 \mathbb{Z}_{+}, \Phi \cap\left\{\alpha_{\ell-1}, \alpha_{\ell}\right\}=\emptyset$;

(5) $\mathfrak{g}$ is of type EIII and $\Phi \subset\left\{\alpha_{3}, \alpha_{4}, \alpha_{5}\right\}=\mathcal{R} \bullet \cap \mathcal{B}$;

(6) $\mathfrak{g}$ is of type FII and $\Phi \subset\left\{\alpha_{1}, \alpha_{2}\right\}$. 
Note that the condition for $M$ being of finite type in the sense of [4] is explicitly described in terms of $\Phi$ in [1, Theorem 9.1].

Proof. We use the results of [1, $\S 13, \S 14]$. We stress the fact that we are assuming that $M$ is of finite type, so that some choices of $\Phi$ are excluded from our consideration because of [1, Theorem 9.1].

First we prove that if $(2.32)$ holds true, then $\left(\mathfrak{g}, \mathfrak{q}_{\Phi}\right)$ satisfies one of the conditions (1)...(6). If $\left(\mathfrak{g}, \mathfrak{q}_{\Phi}\right)$ is not one of those listed in the statement, in all cases, with the two exceptions of CIIa with $\alpha_{2 p-1} \in \Phi$ and $\Phi \cap\left\{\alpha_{h} \mid\right.$ $h>2 p\} \neq \emptyset$, and FII with $\alpha_{3} \in \Phi$, then $M$ admits a G-equivariant fibration onto one of the manifolds described in [1, Examples 14.1, 14.2, and 14.3]. These are strictly pseudoconvex, hence $M$ does not satisfy the higher order Levi form concavity condition by Lemma 5.4. The two remaining cases will be discussed below, while proving the opposite implications.

We know from [1, Theorem 13.4] that $M$ is essentially pseudoconcave, and hence in particular (7.18) and (2.32) hold, in case one of the following is satisfied:

$\left(1^{\prime}\right)$ case $(1)$

$\left(2^{\prime}\right)$ case (2) with either $\Phi \subset \mathcal{R}_{\bullet}$, or $\Phi \cap \mathcal{R}_{\bullet}=\emptyset$,

$\left(3^{\prime}\right)$ case (3) with either $\Phi \subset\left\{\alpha_{1}, \ldots, \alpha_{2 p-1}\right\}$, or $\Phi \subset\left\{\alpha_{2 p+1}, \ldots, \alpha_{\ell}\right\}$,

$\left(4^{\prime}\right)$ case $(4)$,

$\left(5^{\prime}\right)$ case (5) when either $\alpha_{4} \in \Phi \subset \mathcal{R}_{\bullet}$, or $\Phi=\left\{\alpha_{3}, \alpha_{5}\right\}$;

$\left(6^{\prime}\right)$ case (6) when $\Phi \subset\left\{\alpha_{1}, \alpha_{2}\right\}$.

In all of these situations the subellipticity of $\mathfrak{Z}(M)$ was already proved in [12].

To complete the proof, we need only consider the cases in the list in which $M$ is not essentially pseudoconcave. Next we proceed to a case by case discussion.

AIIIa. In this case $\mathfrak{g} \simeq \mathfrak{s u}(p, q)$ with $2 \leq p<q$. Let $\Phi=\left\{\alpha_{j_{1}}, \ldots, \alpha_{j_{k}}\right\}$. We need to discuss the case where $\Phi \cap\left\{\alpha_{p}, \alpha_{q}\right\}=\emptyset$, but $\Phi$ intersects both $\mathcal{R}$. and its complement $\mathcal{B} \backslash \mathcal{R}$. By the condition that $M$ is of finite type, we know from [1, Theorem 13.4] that $\Phi$ does not contain at the same time a simple root $\alpha_{j}$, with $1 \leq j<p$, and its symmetrical $\alpha_{p+q-j}$. Since $\Phi$ and $\Phi^{\prime}=\left\{\alpha_{p+q-j} \mid \alpha_{j} \in \Phi\right\}$ define anti-isomorphic $C R$ manifolds, we can assume in the proof that

$$
\begin{gathered}
1 \leq j_{1}<\cdots<j_{a}<p<j_{a+1}<\cdots<j_{b}<q<j_{b+1}<\cdots<j_{k}<j_{k+1}=p+q, \\
p-j_{a}<j_{b+1}-q .
\end{gathered}
$$

The real roots that correspond to non zero semidefinite Levi forms are

$$
\gamma_{s}=\sum_{j=s}^{p+q-s} \alpha_{j} \quad \text { for } \quad p+q-j_{b+1}<s \leq j_{a} .
$$

Indeed, those $\alpha \in \overline{\mathcal{Q}}_{\Phi} \backslash \mathcal{Q}_{\Phi}$ for which $\mathfrak{g}_{\alpha}^{\mathbb{C}}$ is not contained in the kernel of the Levi form $\mathbf{L}_{\gamma}$ must satisfy $\operatorname{supp}(\alpha) \cap \Phi=\operatorname{supp}(\gamma) \cap \Phi$.

Thus all roots $\alpha$ in $\overline{\mathcal{Q}}_{\Phi} \backslash \mathcal{Q}_{\Phi}$ with $\operatorname{supp}(\alpha) \cap \Phi \not \supset\left\{\alpha_{j_{a}}, \alpha_{j_{b}}\right\}$ belong to $\overline{\mathcal{K}}_{\Phi}$. This is the case in particular for all the simple roots not in $\mathcal{Q}$ and for the roots $-\sum_{j=p}^{q-1} \alpha_{j}$ and $-\sum_{j=p+1}^{q} \alpha_{j}$, whose conjugates are $-\alpha_{q}$ and $-\alpha_{p}$, 
respectively. This implies that all roots in $-\left(\mathcal{Q}_{\Phi}^{n} \cap \overline{\mathcal{Q}}_{\Phi}^{n}\right)$ are sums of roots in $\mathcal{K}_{\Phi} \cup \overline{\mathcal{K}}_{\Phi}$, giving condition (7.18).

CIIa. We need to consider the cases where $\Phi=\left\{\alpha_{j_{1}}, \ldots, \alpha_{j_{k}}\right\}$ contains at the same time a root $\alpha_{j}$ with $j<2 p$ and a root $\alpha_{j}$ with $j>2 p$. This means that $k \geq 2$, and we can order the indices in such a way that

$$
1 \leq \cdots<j_{a}<2 p<j_{a+1}<\cdots \leq \ell,
$$

with $j_{r}$ odd for $1 \leq r \leq a$. The positive real roots are

$$
\gamma_{r}=\alpha_{2 r-1}+\alpha_{\ell}+2 \sum_{j=2 r}^{\ell-1} \alpha_{j} .
$$

Since their supports contain $\alpha_{j_{a+1}}$, they all belong to $\mathcal{Q}_{\Phi}^{n} \cap \overline{\mathcal{Q}}_{\Phi}^{n}$. Let $j_{a}=$ $2 q-1$. The Levi forms $\mathbf{L}_{\gamma_{r}}$ are indefinite if $r>q$ by [1, (ii), p.520]. Next we note that $\mathbf{L}_{\gamma_{r}}$ with $1 \leq r<q$ are identically zero, because there is no root $\alpha$ in $\overline{\mathcal{Q}}_{\Phi} \backslash \mathcal{Q}_{\Phi}$ with $\operatorname{supp}(\alpha) \cap \Phi=\operatorname{supp}\left(\gamma_{r}\right) \cap \Phi$. Indeed, if $\alpha$ is a negative root whose support contains $\operatorname{supp}\left(\gamma_{r}\right) \cap \Phi$ for some $1 \leq r<q$, then $\alpha_{2 q-2}$ and $\alpha_{2 q}$ both belong to $\operatorname{supp}(\alpha)$ and hence also to $\operatorname{supp}(\bar{\alpha})$. This implies that also $\alpha_{2 q-1} \in \Phi$ belongs to the support of both $\alpha$ and $\bar{\alpha}$, showing that $-\alpha \in \mathcal{Q}_{\Phi}^{n} \cap \overline{\mathcal{Q}}_{\Phi}^{n}$.

It was shown in [1, p.520, (iii)] that $\mathbf{L}_{\gamma_{q}}$ is not zero and is positive semidefinite. The set of pairs $\beta, \beta^{\prime} \in \overline{\mathcal{Q}}_{\Phi} \backslash \mathcal{Q}_{\Phi}$ with $\beta+\bar{\beta}^{\prime}=\gamma_{q}$ was shown in [1, p.520, (iii)] to consist of the pairs $\left(\beta_{s}, \beta_{s}\right)$ with

$$
\bar{\beta}_{s}=-\sum_{j=2 q}^{s} \alpha_{j} \quad \text { for } \quad 2 p \leq s<j_{a+1} .
$$

We now distinguish two cases. If $q<p$, the root $-\bar{\alpha}_{2 p}$ belongs to $\mathcal{K}_{\Phi}$. Then the root system generated by $\mathcal{K}_{\Phi} \cup \overline{\mathcal{K}}_{\Phi}$ contains $-\left(\mathcal{Q}_{\Phi}^{n} \cap \overline{\mathcal{Q}}_{\Phi}^{n}\right)$ and hence the higher order Levi form concavity condition (7.18) is satisfied.

When $p=q$, then no element of $\left(\mathcal{K}_{\Phi} \cup \overline{\mathcal{K}}_{\Phi}\right) \backslash \mathcal{R}^{+}$contains $\alpha_{2 p}$ in its support. Thus condition (7.18) fails for $\gamma_{q}$ because, in the expression for $\left(-\gamma_{q}\right)$ in (17.3), the coefficient of $\alpha_{2 p}$ is $(-2)$, while for all roots in $\overline{\mathcal{Q}}_{\Phi}$ it is $\geq(-1)$.

EIII. We only need to consider the cases where either $\Phi=\left\{\alpha_{3}\right\}$, or $\Phi=\left\{\alpha_{5}\right\}$. Due to the symmetry of the EIII diagram, we can restrict our attention to the case where $\Phi=\left\{\alpha_{3}\right\}$.

We note that $\gamma=\alpha_{1}+2 \alpha_{2}+2 \alpha_{3}+3 \alpha_{4}+2 \alpha_{5}+\alpha_{6}$ is the unique positive real root. It belongs to $\mathcal{Q}_{\Phi}^{n} \cap \overline{\mathcal{Q}}_{\Phi}^{n}$ and the corresponding Levi form $\mathbf{L}_{\gamma}$ is semidefinite, having rank 1. Indeed, $\alpha=-\alpha_{1}-\alpha_{2}-2 \alpha_{3}-2 \alpha_{4}-\alpha_{5}$ is the unique root in $\overline{\mathcal{Q}}_{\Phi} \backslash \mathcal{Q}_{\Phi}$ for which $\alpha+\bar{\alpha}=-\gamma$, and there is no other pair $\beta, \beta^{\prime} \in \overline{\mathcal{Q}}_{\Phi} \backslash \mathcal{Q}_{\Phi}$ for which $\beta+\bar{\beta}^{\prime}=-\gamma$. Thus all simple roots belong to $\mathcal{K}_{\Phi} \cup \overline{\mathcal{K}}_{\Phi}$, and hence the higher Levi form concavity condition (7.18) is satisfied.

FII. We are left to discuss the case where $\left\{\alpha_{3}\right\} \subset \Phi \subset\left\{\alpha_{1}, \alpha_{2}, \alpha_{3}\right\}$. There is only one positive real root, namely $\gamma=\alpha_{1}+2 \alpha_{2}+3 \alpha_{3}+2 \alpha_{4}$, that belongs to $\mathcal{Q}_{\Phi}^{n} \cap \overline{\mathcal{Q}}_{\Phi}^{n}$. The corresponding Levi form $\mathbf{L}_{\gamma}$ has rank one, and hence it is semidefinite. The set $\mathcal{K}_{\Phi}$ is the complement of $\left\{-\alpha_{4}\right\}$ in $\mathcal{Q}_{\Phi}$. Then no 
element of $\left(\mathcal{K}_{\Phi} \cup \overline{\mathcal{K}}_{\Phi}\right) \backslash \mathcal{R}^{+}$contains $\alpha_{4}$ in its support. Thus condition (7.18) fails for $\gamma$ because, in the expression for $(-\gamma)$ in (17.3), the coefficient of $\alpha_{4}$ is $(-2)$, while for all roots in $\overline{\mathcal{Q}}_{\Phi}$ it is $\geq(-1)$.

\section{REFERENCES}

[1] A. Altomani, C. Medori, and M. Nacinovich, The CR structure of minimal orbits in complex flag manifolds, J. Lie Theory 16 (2006), no. 3, 483-530. MR MR2248142 (2007c:32043)

[2] Orbits of real forms in complex flag manifolds, Ann. Scuola Norm. Sup. Pisa Cl. Sci. (to appear), preprint, arXiv:math/0611755.

[3] S. Araki, On root systems and an infinitesimal classification of irreducible symmetric spaces, J. Math. Osaka City Univ 13 (1962), 1-34.

[4] T. Bloom and I. Graham, A geometric characterization of points of type $m$ on real submanifolds of $\mathbf{C}^{n}$, J. Differential Geometry 12 (1977), no. 2, 171-182. MR MR0492369 (58 \#11495)

[5] N. Bourbaki, Éléments de mathématique, Hermann, Paris, 1975, Fasc. XXXVIII: Groupes et algèbres de Lie. Chapitre VII: Sous-algèbres de Cartan, éléments réguliers. Chapitre VIII: Algèbres de Lie semi-simples déployées, Actualités Scientifiques et Industrielles, No. 1364. MR MR0453824 (56 \#12077)

[6] A. Bove, M. Derridj, J. J. Kohn, and D. S. Tartakoff, Sums of squares of complex vector fields and (analytic-) hypoellipticity, Math. Res. Lett. 13 (2006), no. 5-6, 683701. MR MR2280767 (2007k:35051)

[7] M. Christ, A remark on sums of squares of complex vector fields, 2005.

[8] C. Fefferman and D. H. Phong, The uncertainty principle and sharp Gärding inequalities, Comm. Pure Appl. Math. 34 (1981), no. 3, 285-331. MR MR611747 (82j:35140)

[9] E. Hebey, Sobolev spaces on Riemannian manifolds, Lecture Notes in Mathematics, vol. 1635, Springer-Verlag, Berlin, 1996. MR MR1481970 (98k:46049)

[10] S. Helgason, Differential geometry, Lie groups, and symmetric spaces, Pure and Applied Mathematics, vol. 80, Academic Press, New York, 1978. MR MR514561 (80k:53081)

[11] C. D. Hill and M. Nacinovich, Pseudoconcave $C R$ manifolds, Complex Analysis and Geometry (New York) (V. Ancona, E. Ballico, and A. Silva, eds.), Lecture Notes in Pure and Applied Mathematics, vol. 173, Marcel Dekker, Inc, 1996, pp. 275-297.

$[12] \ldots$, A weak pseudoconcavity condition for abstract almost $C R$ manifolds, Invent. Math. 142 (2000), 251-283.

[13] Weak pseudoconcavity and the maximum modulus principle, Ann. Mat. Pura Appl. (4) 182 (2003), no. 1, 103-112. MR MR1970466 (2004k:32056)

[14] L. Hörmander, Hypoelliptic second order differential equations, Acta Math. 119 (1967), 147-171. MR MR0222474 (36 \#5526)

[15] $\longrightarrow$, The analysis of linear partial differential operators. III, Grundlehren der Mathematischen Wissenschaften [Fundamental Principles of Mathematical Sciences], vol. 274, Springer-Verlag, Berlin, 1985, Pseudodifferential operators. MR MR781536 (87d:35002a)

[16] J. J. Kohn, Pseudo-differential operators and hypoellipticity, Partial differential equations (Proc. Sympos. Pure Math., Vol. XXIII, Univ. California, Berkeley, Calif., 1971), Amer. Math. Soc., Providence, R.I., 1973, pp. 61-69. MR MR0338592 (49 \#3356)

[17] Multiplier ideals and microlocalization, Lectures on partial differential equations, New Stud. Adv. Math., vol. 2, Int. Press, Somerville, MA, 2003, pp. 141-151. MR MR2055843 (2005a:35050)

[18] - Hypoellipticity and loss of derivatives, Ann. of Math. (2) 162 (2005), no. 2, 943-986, With an appendix by Makhlouf Derridj and David S. Tartakoff. MR MR2183286 (2006k:35036)

[19] J. J. Kohn and L. Nirenberg, Non-coercive boundary value problems, Comm. Pure Appl. Math. 18 (1965), 443-492. MR MR0181815 (31 \#6041) 
[20] C. Medori and M. Nacinovich, Algebras of infinitesimal CR automorphisms, J. Algebra 287 (2005), no. 1, 234-274. MR MR2134266 (2006a:32043)

[21] M. Nacinovich, On weakly pseudoconcave CR manifolds, Hyperbolic problems and regularity questions, Trends Math., Birkhäuser, Basel, 2007, pp. 137-150. MR MR2298789 (2008d:32034)

[22] C. Parenti and A. Parmeggiani, On the hypoellipticity with a big loss of derivatives, Kyushu J. Math. 59 (2005), no. 1, 155-230. MR MR2134059 (2005m:35049)

[23] _ A note on Kohn's and Christ's examples, Hyperbolic problems and regularity questions, Trends Math., Birkhäuser, Basel, 2007, pp. 151-158. MR MR2298790 (2007m:35019)

[24] J. A. Wolf, The action of a real semisimple group on a complex flag manifold. I. Orbit structure and holomorphic arc components, Bull. Amer. Math. Soc. 75 (1969), 1121-1237.

A. Altomani: Research Unity in Mathematics, University of Luxembourg, 162A, AVEnue de la Fä̈encerie, L-1511 LuxembourG

E-mail address: andrea.altomani@uni.lu

C.D.Hill: Department of Mathematics, Stony Brook University, Stony Brook, NY 11794, USA

E-mail address: dhill@math.sunysb.edu

M. Nacinovich: Dipartimento di Matematica, II Università di Roma "Tor Vergata", Via della Ricerca Scientifica, 00133 Roma, Italy

E-mail address: nacinovi@mat.uniroma2.it

E. Porten: Department of Mathematics, Mid Sweden University, 85170 SundSVALL, SwEDEN

E-mail address: Egmont.Porten@miun.se 